\title{
PROBLÈMES \\ D'ACCUMULATION DANS \\ LE LIT \\ DU DANUBE \\ ENTRE DEVIN ET SZOB
}

\author{
PAR \\ J. FURDICK * \\ ET \\ K. STELCZER ${ }^{* *}$
}

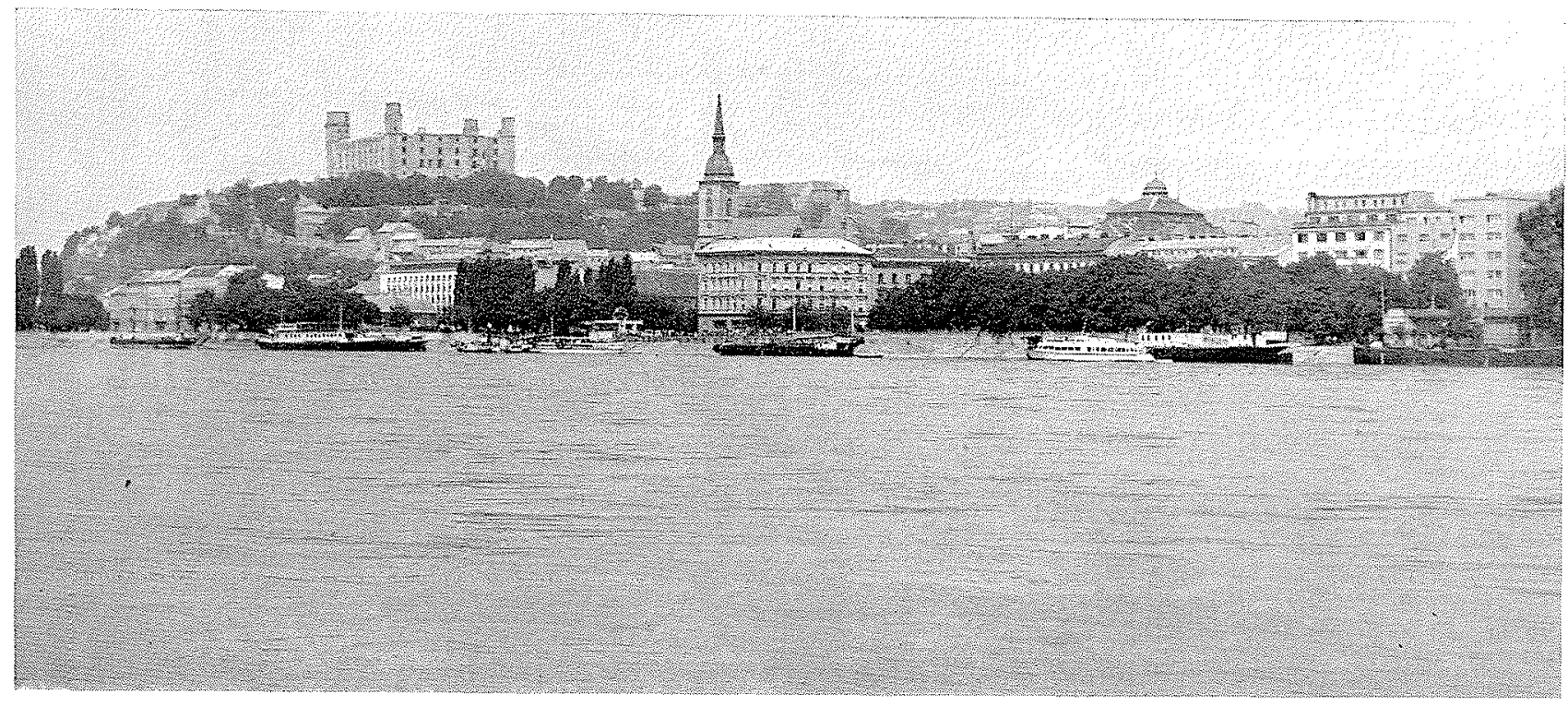

Un panorama extraordinaire et inattendu s'offre aux yeux du navigateur sur le Danube lorsqu'il entre, par la «Porte de Devin », dans le bassin des Karpates. Il voit encore les collines de Bratislava avec les ruines pittoresques du château (fig. 1), mais le Danube se divise déjà en de nombreuses branches et s'étale d'une manière bien caractéristique du cours inférieur des fleuves, créant une multitude d'îles et îlots sur le grand cône de déjection entre

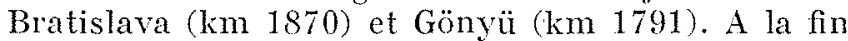
du tertiaire, il y avait ici un lac intérieur, qui fut remblayé en premier lieu par le débit solide du

* Ingénieur, Directeur de l'Institut de Recherches de l'Economie Hydraulique à Bratislava (Tehécoslovaquie).

* Ingénieur, Directeur de l'Institut de Recherches des Ressources Hydrauliques a Budapest (Hongrie).
Danube. Par suite du dépôt continuel du débit solide, la partie du cône de déjection située entre les levées de protection ne cesse de s'exhausser, même aujourd'hui. L'importance du remblayage est illustrée par le fait que l'épaisseur de la couche de gravier alluvionnaire dépasse $400 \mathrm{~m}$, voire même $2500 \mathrm{~m}$ en certains endroits. Et pour que le contraste soit plus marqué, ce secteur du Danube étalé sur la plaine alluviale (fig. 2) a été nommé le «Haut-Danube». En effet, c'est la partie supérieure du secteur central, au point de vue géographique, du Danube (Devin-Portes de Fer). C'est un secteur de transition entre le cours supérieur et le secteur à caractère de plaine, séparés l'un de l'autre par une rupture de pente géologique. Alors que sur le secteur Bratislava-Palkovičovo $(\mathrm{km} \mathrm{1810)}$ la pente moyenne est de $35-40 \mathrm{~cm}$ par $\mathrm{km}$, après 


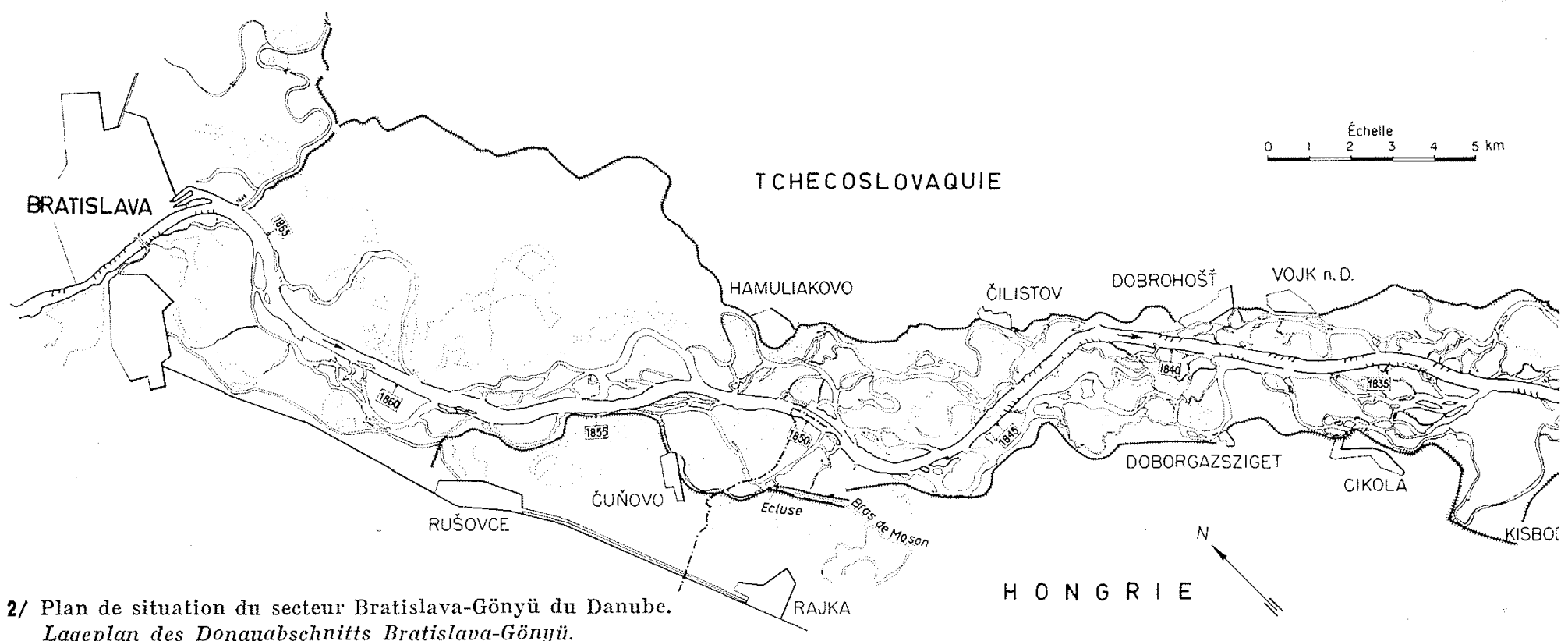

Lageplan des Donauabschnitts Bratislava-Gönyii.
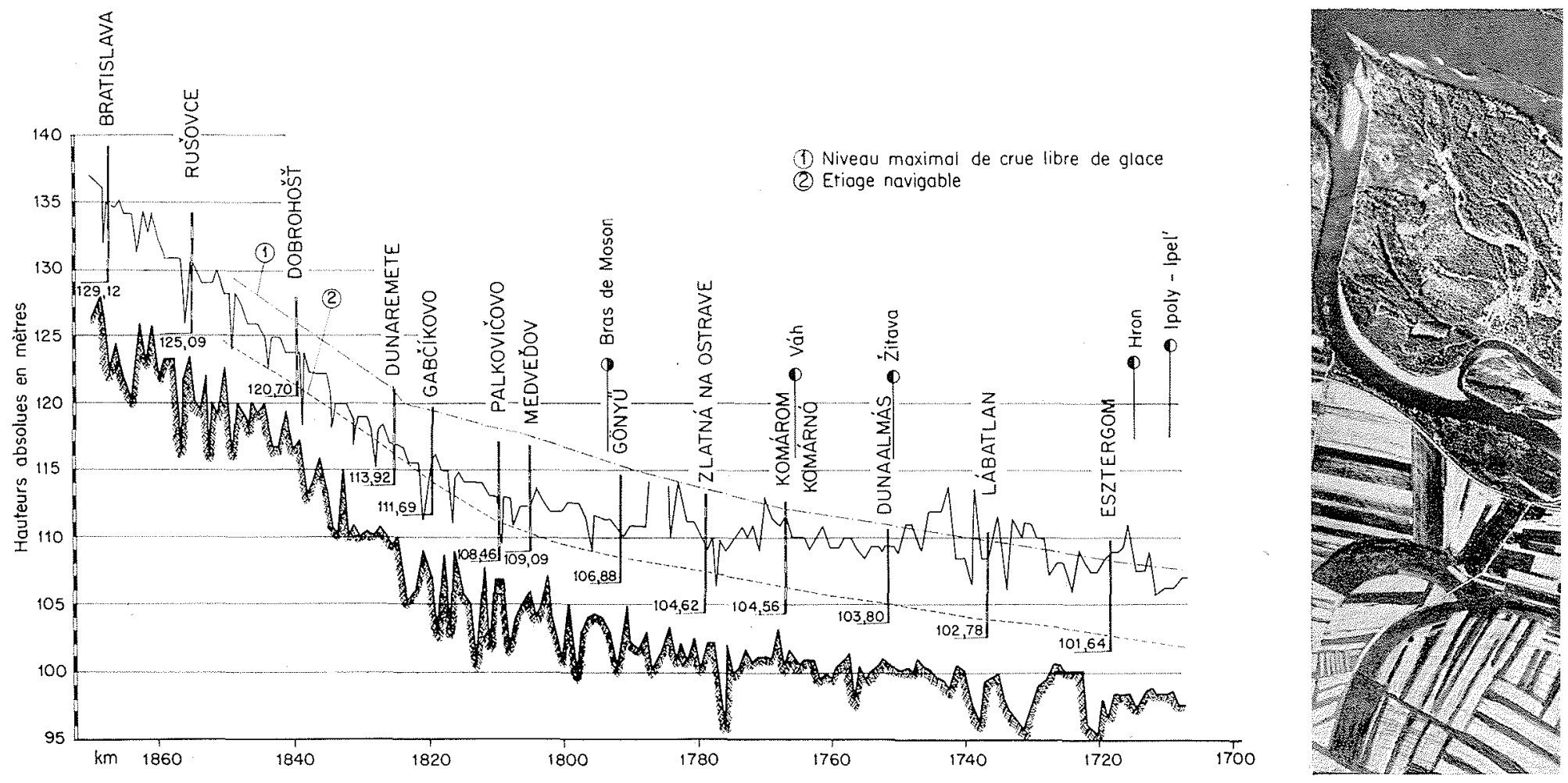

3/ Profil en long schématique du secteur du Danube Schematisches Längsprofil des Donalabschnitts zwischen entre Bratislava et l'embouchure de l'Ipel' - Ipoly. Bratislana and der Ipelmiundung (Ipoly).

le court secteur de transition Palkovičovo-Gönyü ( $\mathrm{km} \mathrm{1791),} \mathrm{avec} \mathrm{une} \mathrm{pente} \mathrm{de} 12$ à $15 \mathrm{~cm}$ par $\mathrm{km}$, cette valeur tombe, en aval de Gönyü, à $6-10 \mathrm{~cm} / \mathrm{km}$ (fig. 3).

\section{Conditions hydrologiques.}

RÉGHE DES EAUX: LES DÉBITS :

Le caractère du régime d'eau et les conditions d'écoulement du secteur considéré du Danube sont grandement influencés par le réseau de bras secondaires, le sous-sol alluvial fort perméable et la rupture de pente au $\mathrm{km} 1810$.
Pendant les ondes de crue, dans la période de montée, les eaux remplissent les bras secondaires, le lit majeur et le sous-sol; une partie de l'eau arrivant à ce secteur s'emmagasine. Pendant la période de décrue, l'eau emmagasinée s'écoule en partie. Sous l'action de ces phénomènes, le régime du secteur aval est plus équilibré que celui du secteur d'amont.

Le passage des ondes de crue est encore influencé par la rupture de la pente à Palkovičovo. A l'aval de ce point, leur vitesse de propagation diminue et l'eau s'étale. Une nouvelle onde de crue peut raltraper la précédente, qui a déjà rempli le lit, 


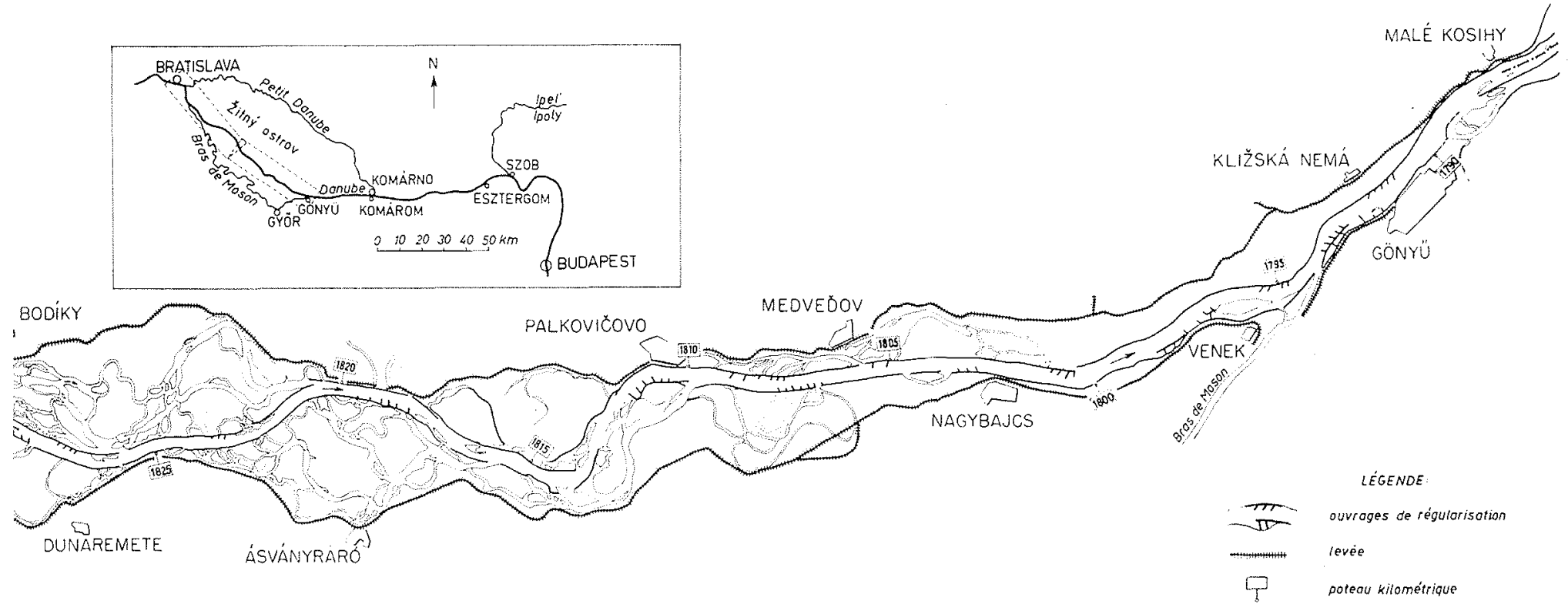

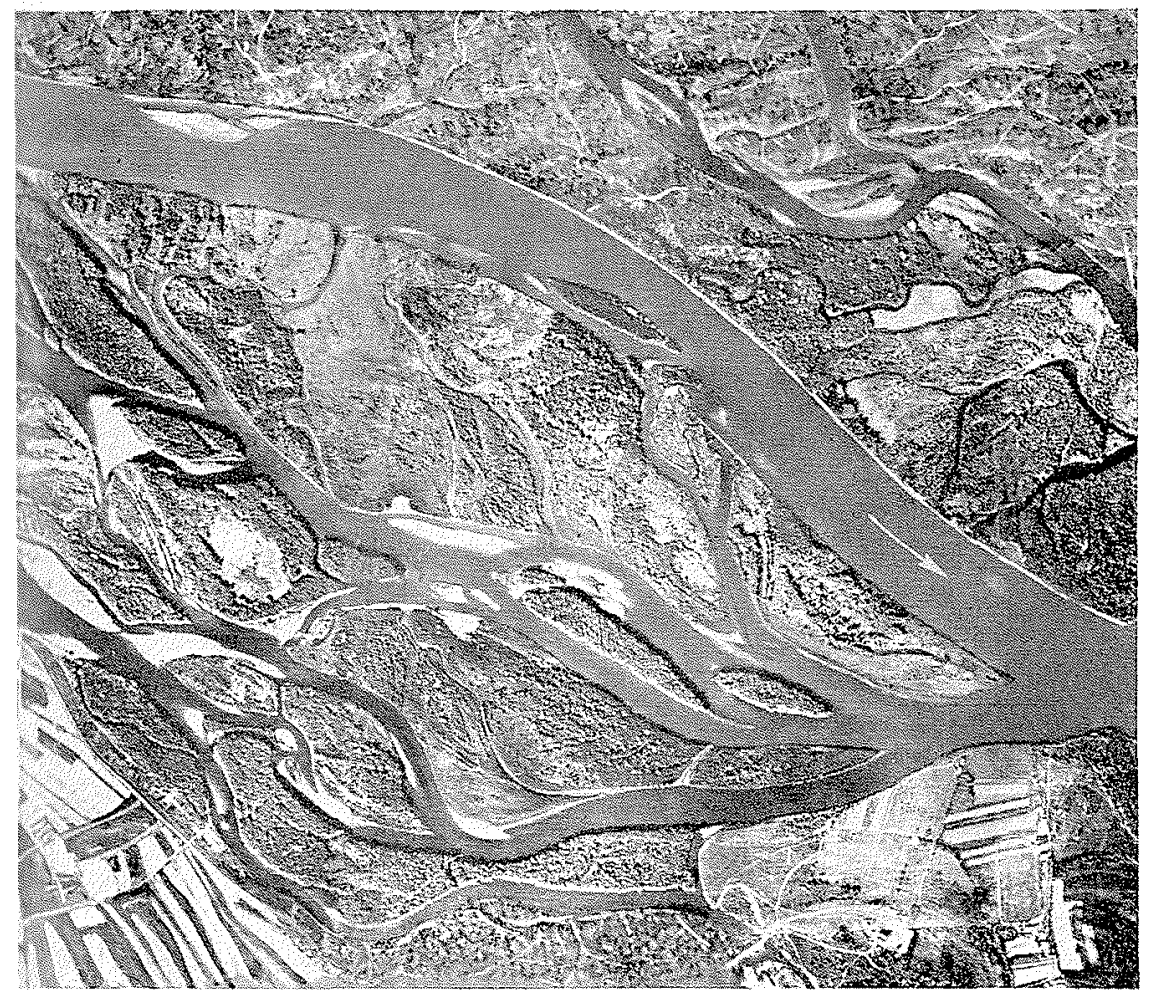

4/ Photographie aérieme d'un secteur du « Haut-Danube». Luftaunahme eines Abschnitts der «Oberen Donau».

et une situation dangereuse peut se produire au point de vue de la défense contre les crues.

A l'aval de Bratislava, le transport d'eau du lit principal diminue sous l'efret de drainage et d'emmagasinement des nombreux bras secondaires, puis à l'aval de la rentrée de ces bras, à Medvedov ( $\mathrm{km}$ 1806), leur débit rejoint dans sa presque tota. lité le lit principal (fig. 3).

Du point de vue du débit du lit principal, on peut distinguer quatre étapes caractéristiques, à savoir :

a) Le débit total s'écoule dans le lit principal (jusqu'au débit de $1800 \mathrm{~m}^{3} / \mathrm{s}$ à Bratislara); b) L'eau se déverse par-dessus les ouvrages de fermeture des bras secondaires et remplit progressivement leurs lits (débit de $1800-2300 \mathrm{~m}^{3} / \mathrm{s}$ मे Bratislava);

c) L'eau atteint les crêtes des rives, réunit le lit principal et les bras secondaires en un système actif de transport d'eau (débit voisin de $4000 \mathrm{~m}^{3} / \mathrm{s}$ à Bratislava);

d) L'eau remplit le lit majeur tout entier (débit de $4000-6000 \mathrm{~m}^{3} / \mathrm{s}$ à Bratislava).

A l'époque de crue, les quatre étapes se reconvrent les unes les autres.

Le tableau I montre les débits du lit principal dans les différents profils du secteur considéréé, en pourcentage du débit de Bratislava.

Des données du tableau, on peut voir que, pour des apports supérieurs à $2000 \mathrm{~m}^{3} / \mathrm{s}$, le débit du lit principal varie beaucoup de secteur en secteur. Par conséquent, chaque secteur a ses conditions d'écoulement caractéristiques différentes suivant l'importance et la capacité des systèmes de bras secondaires.

Nous donnons dans le tableau II les débits caractéristiques du secteur examiné.

Les données du tableau confirment le bien-fondé des constatations que nous avons faites par rapport au régime d'eau du secteur examiné du Danube, à savoir que, jusqu'à Gönyü, les débits de crue diminuent sous l'effet des bras secondaires, de la largeur du champ d'inondation et du sous-sol perméable, tandis que les débits moyens et minimaux augmentent.

Conditrons de pentes (fig. 2):

Entre les $\mathrm{km} 1870$ et $\mathrm{km} 1840$ la pente est presque constante : $0,35-0,37 \%$.

En aval du km 1840 jusqu'au km 1821, la pente moyenne diminue lentement $(0,28-0,30 \%)$, puis du $\mathrm{km} 1821$ au $\mathrm{km} 1805$, plus rapidement $(0,18-0,20 \%)$.

Sur le secteur allant du $\mathrm{km} 1805$ au $\mathrm{km} \mathrm{1770,}$ la diminution est de nouveau ralentie : la pente tombe de $0,18-0,10$ successivement jusqu'à 0,10 $0,06 \%$. Les pentes générales sont toutefois forle ment influencées par les conditions locales.

Les valeurs limites locales atteignent respectim 


\section{J. FURDICK ET K. STELCZER}

vement $0,43 \%$ et $0,05 \%$. Par suite de l'influence des bras secondaires, il ne se rencontre presque aucun secteur dans lequel la pente soit à peu près constante. Les variations minimales se présentent sur les secteurs allant du $\mathrm{km} 1830$ au $\mathrm{km} \mathrm{1814,}$ du $\mathrm{km} 1805$ aul $\mathrm{km} 1795$, et du $\mathrm{km} 1785$ au

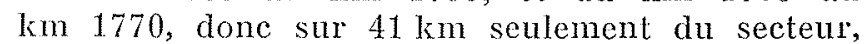
long de $100 \mathrm{~km}$. Les secteurs où la pente diminue si le débit augmente méritent de retenir une attention spéciale, tels sont les secteur's du km 18591860 , des kilomètres 1854-1855, 1842-1843, 1832$1834,1828-1830,1821-1822,1817-1819,1810-1814$ et 1807-1808.

RÉGME DES GLACES :

L'apparition de la glace sur le Danube est un phénomène se répétant chaque année (fig. 5).

Les conditions des glaces du secteur examiné sont caractérisées par le fait que les embâcles provoquant des dangers se présentent dans les années oì la prise du fleuve se produit en période d'étiage. Les observations de longue durée révèlent les endroits caractéristiques des embâcles. Ceux-ci se situent sur les hauts-fonds se formant à l'aval des grandes courbes, les plus caractéristiques d'entre eux se produisant dans le secteur à l'aval de Bratislava et au voisinage des kilomètres 1836 à 1838 .

Au cas où la prise a lieu en période d'étiage, un phénomène intéressant se poursuit sur le secteur du $\mathrm{km} 1850$ au $\mathrm{km} \mathrm{1816.} \mathrm{Le} \mathrm{niveau} \mathrm{d'eau} \mathrm{du} \mathrm{fleuve}$ est plus bas que le niveau de la nappe phréatique; de ce fait, les eaux souterraines, de température relativement élevée, font diminuer dans les bras secondaires l'épaisseur de la glace, et même la font fondre dans certains cas.

LE LIT DU FLEUVE:

Les dimensions géométriques du lit sont déterminées par la pente du fleuve, les matériaux constituant le lit et les débits liquide et solide à transporter. Si les dimensions du lit varient considérablement dans l'état naturel, c'est par la régularisation qu'il faut assurer leur variation graduelle et uniforme. En ce qui concerne le secteur dz: Danube considéré, c'est la variation excessive des dimensions du lit qui l'ont caractérisé avant la régularisation. Le fleuve divaguant sur son cône de déjection en matériaux meubles a changé son lit après chaque crue (fig. 6).

Au cours de la régularisation, un lit principal a été créé; cependant, le fond du lit n'a pas cessé d'avoir une tendance à s'exhausser. La figure 7 montre, pour chaque échelle limnimétrique du secteur, les hauteurs d'eau correspondant, dans les années 1920 à 1960 , à la hauteur d'eau de $+300 \mathrm{~cm}$ à Bratislava par rapport au niveau de l'étiage navigable fixé en 1932.

La figure indique nettement le caractère d'exhaussement de ce secteur.

En examinant la figure 8 , on peut constater que le plus grand exhaussement du niveau d'étiage, par rapport au niveau d'étiage navigable fixé en 1934 (niveau correspondant au débit de $1000 \mathrm{~m}^{3} / \mathrm{s}$ ), s'est produit entre les kilomètres 1820 (Gabčikovo) et 1813 .

Dans le secteur allant du $\mathrm{km} \quad 1826,63$ au km 1823,78 , l'exhaussement annuel est de $2,72 \mathrm{~cm}$, au $\mathrm{km} 1820$, de $2,52 \mathrm{~cm}$, et au $\mathrm{km} 1810$, elle est de $2,40 \mathrm{~cm}$. Pendant les années 1951-1959, une certaine stabilisation a eu lieu aux environs des échelles de Dobrohošt (km 1840), Gabčikovo (km 1820) ef Gönyü (km 1791), et même un creusement partiel

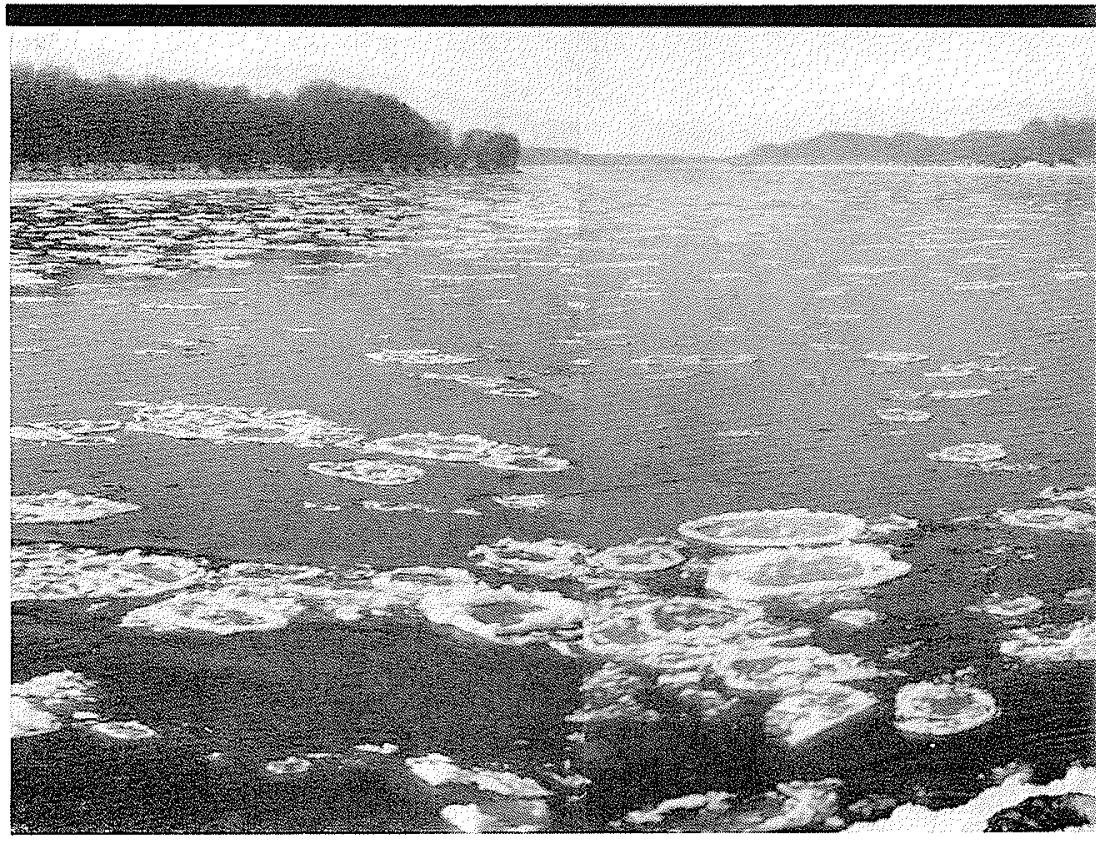

5/ Photo d'un secteur du Danube. Les premiers glaçons.

Aufnahme eines Donauabschnitts. Die ersten Eisschollen.

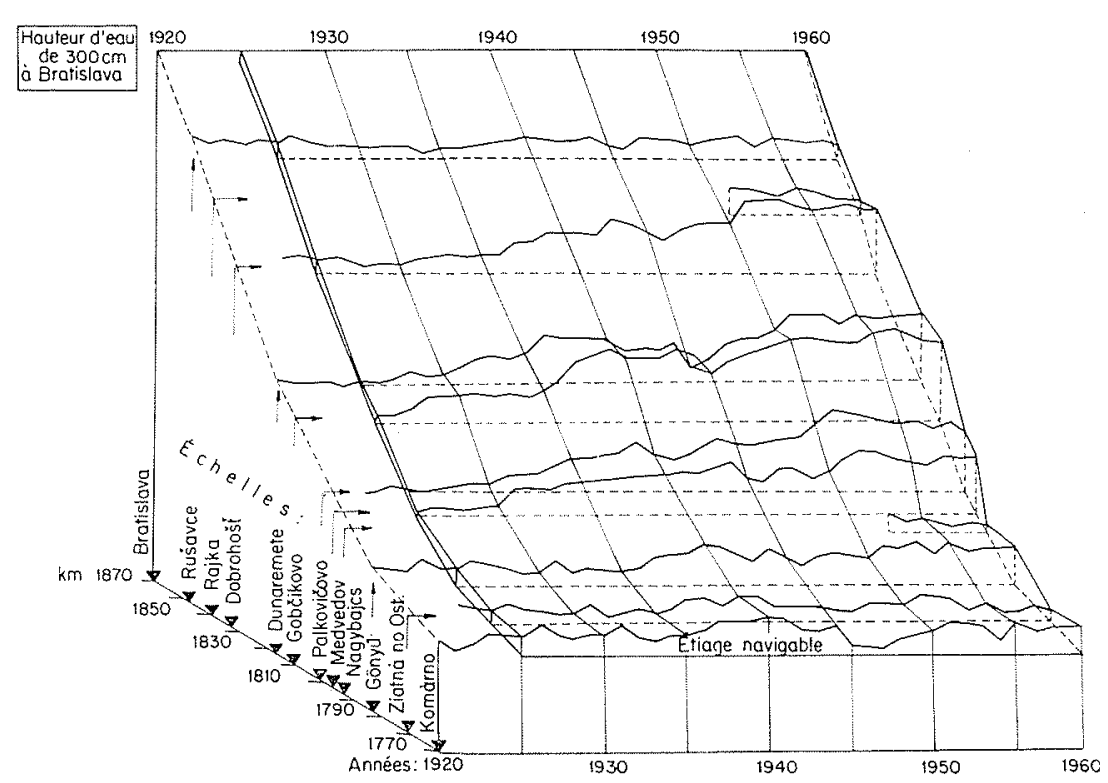

1/ Variation, au cours des années 1920-1960, des niveaux d'eau correspondant aux échelles du secteur Bratislava-Komámo du Danube à la hauteur d'eau de $+300 \mathrm{~cm}$ à Bratislava.

Schwankungen der Wasserstände, die sich auf die Pegel im Donauabschnitt Bratislava-Komarno bei einer Spiegelhöhe von $+300 \mathrm{~cm}$ bei Bratislaba beziehen (1920-1960).

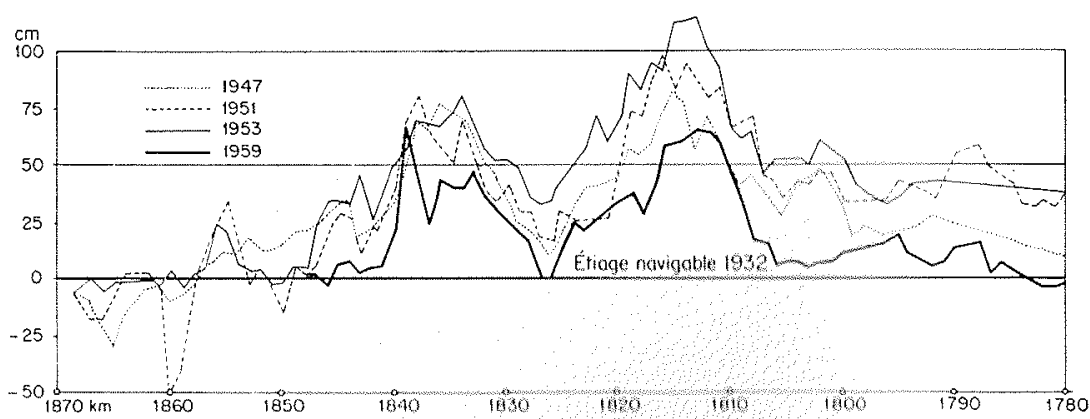

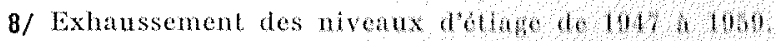

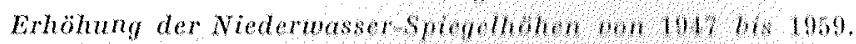



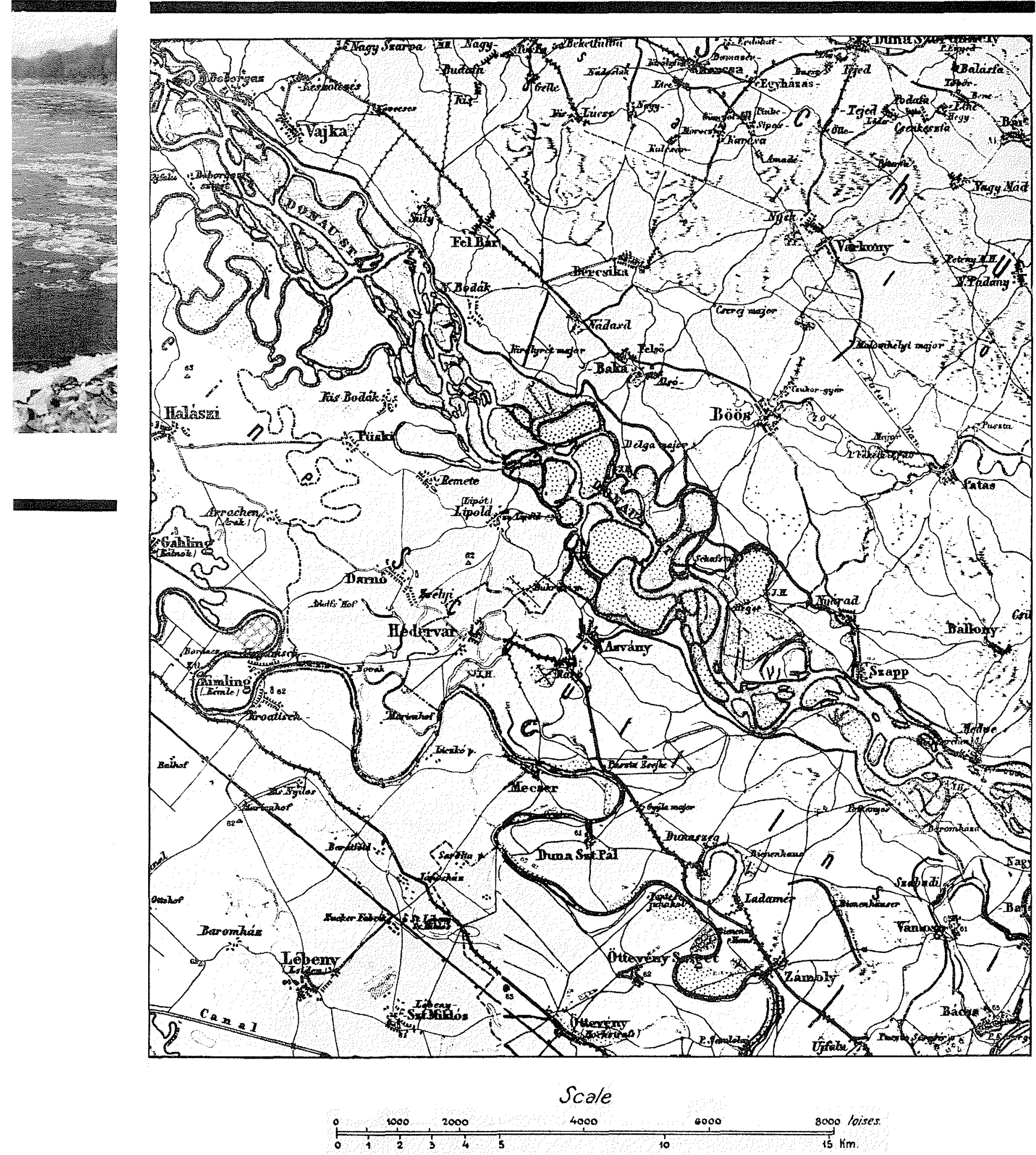

6/ Un secteur du Danube entre Bratislava et Gönyüi avant la régularisation. Détail de la carte topographique de l'époque.

Ein Donauabschnitt zwischen Bratislava und Gönyü vor der Regulierung. Auszug aus der topographischen Karte dieser Zeit. 
aux environs des échelles de Palkovičovo ( $\mathrm{km} \mathrm{1810),}$ de Medvedov (km 1806) et de Zlatna na $O$. ( $k m$ 1778). Ces phénomènes peuvent être expliquẻs par le dragage intensif effectué pendant la période examinée.

La hauteur du terrain du champ d'inondation diminue à partir des rives du lit principal vers les bords du cône de déjection. En effet, les eaux débordantes perdent, par suite de l'étalement, la majeure partie de leur force tractrice, ce qui a pour conséquence une sédimentation au voisinage des rives. Aujourd'hui même, cette chaîne de dépôts sur les rives est une des caractéristiques du profil transversal du lit majeur.

La largeur du plan d'eau varie entre des limites très larges, même pour des hauteurs d'eau iden. tiques. Aux plus hautes eaux, la largeur du plan d'eau dépasse par endroits $5 \mathrm{~km}$, entre les berges naturelles, comme entre les levées de protection contre les crues, alors qu'elle n'atteint pas $1 \mathrm{~km}$ ailleurs.

\section{LA VITESSE DU COURANT :}

Un des éléments hydrauliques les plus variables du secteur est la vitesse de l'eau. Elle est influencée d'une manière décisive, en dehors de la pente et de la hauteur d'eau, par les dimensions du profil transversal. A des conditions moyennes, la vitesse moyenne est de $2,2 \mathrm{~m} / \mathrm{s}$ à l'extrémité amont du secteur, alors qu'à la limite aval c'est la vitesse de $0,9 \mathrm{~m} / \mathrm{s}$ qui est caracléristique.

Sur la partie la plus critique du secteur, entre Rajka et Medvedov (Km 1848-1806), les vitesses moyennes se présentant dans le lit principal sont indiquées dans le tableau III, en fonction du débit à Bratislava.

\section{Transport DE DÉBIT SOLIDE:}

Un charriage extraordinairement intensif est caractéristique du secteur du Danube entre Rajka et Gönyü. La plus grande partie du débit solide venant $d u$ secteur autrichien, soit environ 2,2 millions de mètres cubes par an, est constituée par des matériaux en suspension, alors que le débil charrié se monte à environ $600000 \mathrm{~m}^{3}$ à Rajka. Néanmoins, c'est ce dernier qui joue le rôle décisif, puisque sa plus grande partie se dépose entre les kilomètres 1820 et 1806 par suite de la diminution de la force tractrice, pendant que le débit solide en suspension continue son chemin dans sa totalité, sans entraves. Le mouvement du débit charrié s'effectue selon les lois régies par les conditions hydrauliques et hydrologiques locales, fort variables et s'enlaçant mutuellement les umes aux autres sous l'effet de la ségrégation simultanée et de l'usure des grains des matériaux transportés.

La figure 9 montre le mouvement du débit charrié au fond du lit. On y peul distinguer une partie du fond (A) où les grains ne bougent pas, généralement sur la partie voisine de la berge, du cóté convexe du profil transversal, ou la force tractrice n'atteint que rarement, pendant la période des hautes eaux, sa valeur critique. Les grains peuvent être envasés par des matières fines, ce qui, toutefois, n'arrive généralement guère sur le secteur examiné du Danube. En ce qui concerne la formation du lit, les exhaussements du fond, de caractère local, prédominent à ces endroits.

Une autre partic caractéristique du périmètre est la bande de transition, caractérisée par le mouve- ment de grains individuels. Le mouvement peut s'étendre, en certains cas, sur toute la largeur de la bande du charriage. (Les seuils sont caractérisés en général par un mouvement sur toute la largeur du fond.)

Dans la bande adjacente, le mouvement du débit charrié est intense; des banes d'alluvions, des dunes se produisent. Dans le cas de rivieres aux rives concaves bien formées et de tracé adéquat, la zone

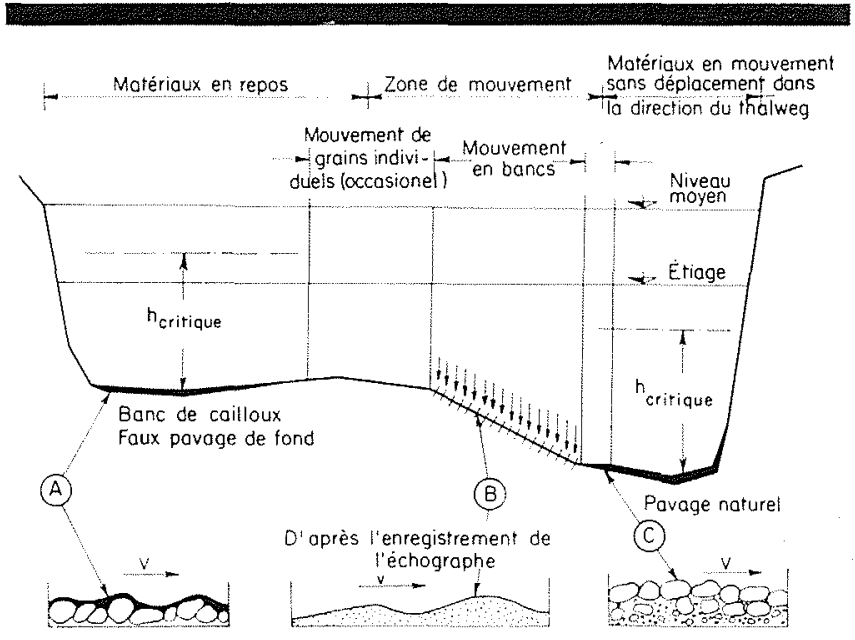

9/ Zones de mouvement du matériau charrié dans un profil transversal.

Bewegungszonen der Geschiebefracht in einem Querprofil.

de charriage du débit solide correspond à la partie $\mathrm{du}$ fond indiquée par $\mathrm{B}$ sur la figure 8 .

Dans la zone C du profil examiné, il n'y a pas charriage dans la direction du thalweg, car c'est la partie du profil transversal où le courant transversal est le plus fort. Par suite de l'entraînement des particules fines, une couche de protection efficace, un "pavage naturel» du fond, se forme ici, ce qui empèche une érosion ultérieure. Les cailloux se déplacent rarement dans cette zone, car le courant transversal rend le passage des grains impossible.

La figure 10 montre la variation du diamètre effectif (à $65 \%$ ) du matérian de fond dans le secteur examiné, sur la base de l'analyse des sept échantillons pris dans chaque profil. Jusqu'au $\mathrm{km} 1822$, une diminution de $0,04 \mathrm{~mm} / \mathrm{km}$ peut être observée, avec des écarts locaux considérables. Du km 1822 au $\mathrm{km} 1810$ le diamètre effectif des grains diminue de $1 \mathrm{~mm} / \mathrm{km}$; en aval du $\mathrm{km} 1810$ jusqu'à Zlatna na $O$. (km 1778), l'usure atteint $0,08 \mathrm{~mm} / \mathrm{km}$ avec des variations minimes. L'écart entre les minimums et les maximums des diamètres effectif's déterminés pour une série de profils transversaux est de $10 \mathrm{~mm}$ dans le premier secteur. de $2 \mathrm{~mm}$ dans le deuxième et de $5 \mathrm{~mm}$ dans le troisième.

A la suite des travaux de régularisation à basses eaux effectués entre les kilomètres 1863 et 1822 (secteur I sur la figure 11), la rugosité du lit est considérablement accrue entre les kilomètres 1822 et 1810 (secteur II); un classement fort accentué du débit charrié a lieu sous l'influence des embranchements (variations subites du débit et de la pente locale). Plus en aval, sur le troisième secteur, les matériaux du fond deviennent plus gros, probablement par suite des travaux de dragage considé- 


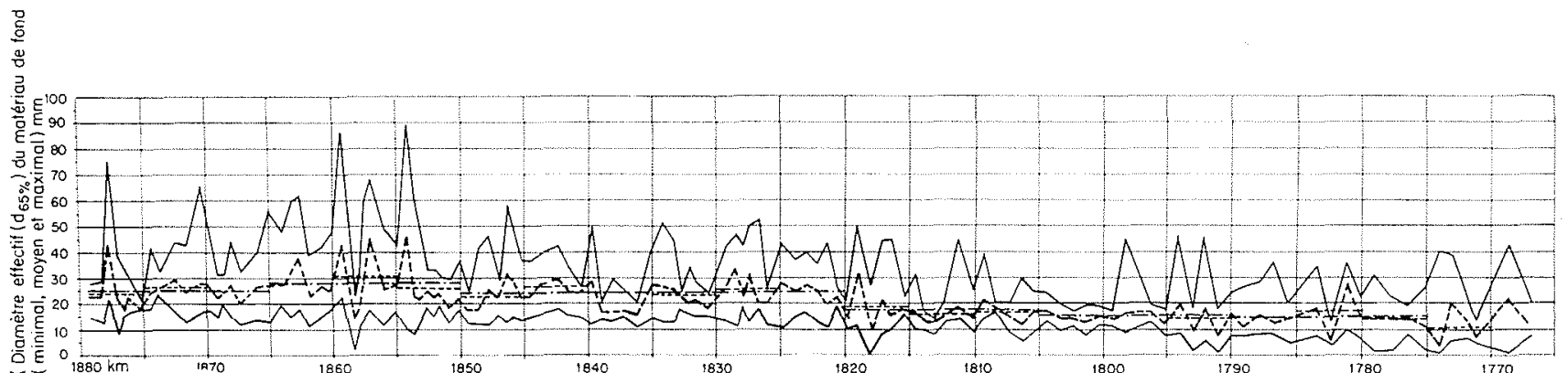

10/ Variations des valeur's moyennes et extrêmes du diamètre effectif ( $d 6 \pi \%)$ des matériaux de fond

Schwankungen der mittleren und Höchstwerte des wirksamen Durchmessers

$(d 6 . \%)$ des Sohlenmaterials.

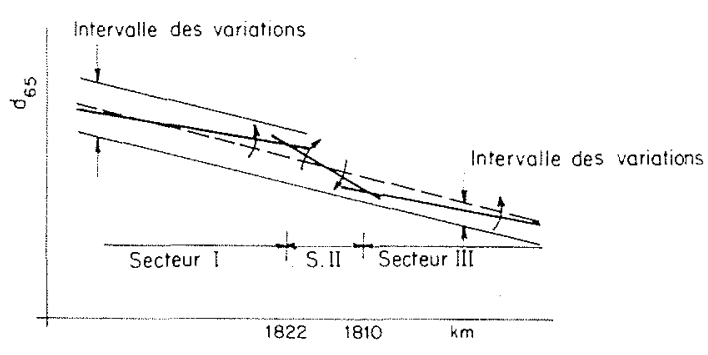

11/ Limites de variation du diamètre effectif ( $d \quad 6 \pi \%)$ du matériau de fond.

Schwanlangsgrenzen des wirlisamen Durchmessers $(d, 0 \%)$ des Sohlenmaterials.

12/ Courbes granulométriques du matériau de fond pour quelques profils choisis. Sieblurven des Sohlenmaterials fïr einige ausgesuchte profile.

rables effectués entre les kilomètres 1780 et plus en aval.

Sur la figure 12 , nous avons représenté les courbes granulométriques du matériau du fond pour trois profils caractéristiques. On voit nettement que la granulométrie du débit charrié devient toujours plus fine.

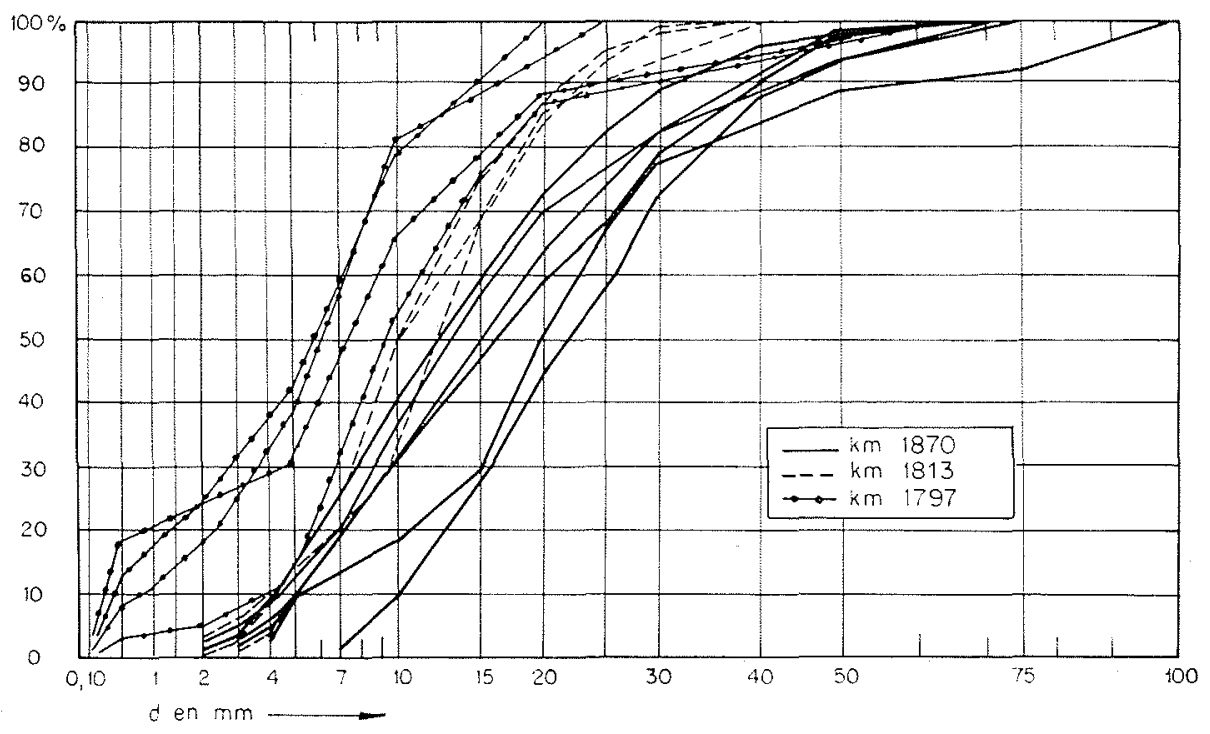

Quant aux matériaux en suspension du secteur considéré, on peut distinguer deux groupes d'orisine différente. Au premier groupe appartiennent les grains fins provenant du bassin versant. Leur concentration ne cesse de varier, même à des cpoques ayant des conditions d'écoulement idenliques, et n'est en aucun rapport avec des caractéristiques hydrauliques.

Le deuxième groupe comprend les grains plus gros, dont la concentration suit les variations du lébit liquide. Cette partie du débit solide en suspension prend part à un processus incessant d'échanges, passant du fond à l'état de suspension et inversement.

Le tableau IV indique les transports moyens annuels du secteur examiné en fonction du débit d'eau moyen annuel.

LES EAUX PHRÉtioUes :

Le Danube, en tant que lit principal de sa vallée, influence, dans une grande mesure, le régime des eaux phréatiques des terrains environnants. Grâco à la perméabilité importante des graviers du cône de déjection (coefficient de perméabilité $k=0,5$ $1 \mathrm{~cm} / \mathrm{s}$ ) et à l'épaisseur considérable des couches 


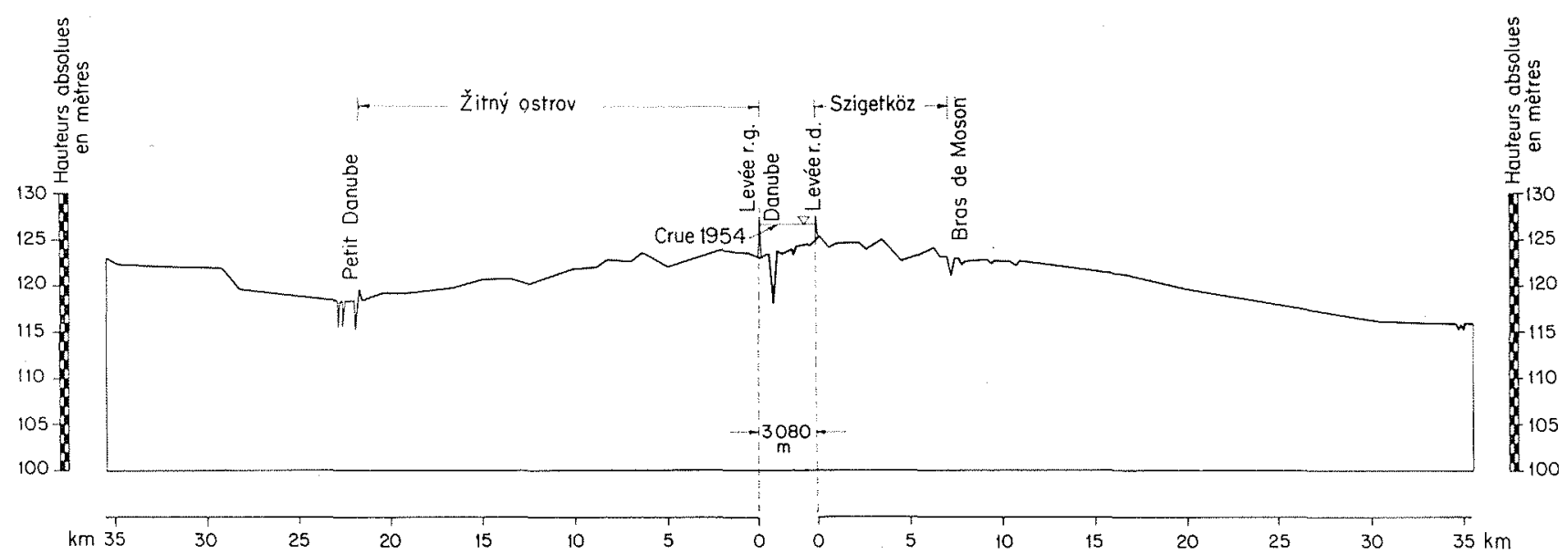

13/ Coupe transversale du cône de déjection du Danube au $\mathrm{km} 1840$. Querschnitt des Schuttkegels der Donau bei $\mathrm{km} 1840$.

perméables sous les levées $(10$ à $200 \mathrm{~m}$ ), les eaux phréatiques des terrains protégés sont en liaison étroite avec les eaux du Danube. Le relèvement de la nappe phréatique suit cependant beaucoup plus rapidement les crues du Danube qu'on ne pourrait le croire en faisant un calcul avec le coefficient «k». La cause en est la poussée hydrostatique, le plan d'eau étant plus haut dans le lit du Danube que le fond de la vallée (fig. 13). La vitesse de propagation de cette pression change, d'après sa grandeur et d'après la perméabilité des couches inférieures, entre 0,3 et $3 \mathrm{~m} / \mathrm{s}$. Dans les cas exceptionnels, elle atteint $5,5 \mathrm{~m} / \mathrm{s}$.

Les variations les plus importantes du niveau de la nappe phréatique se produisent en aval de Bratislava, dans les parties supérieures des deux grandes îles formées du coté gauche par le bras dit Petil Danube et du coté droit par le bras de Moson (Zitny ostrov et Szigetliöz) (voir fig. 2), dans uı troncon long de $40 \mathrm{~km}$ et jusqu'à une distance de $8.10 \mathrm{~km}$ du lit principal. Cette bande active devient progressivement de plus en plus étroite dans la direction du courant.

Le Danube atteint son plus haut niveau au cours des mois de printemps et d'été, c'est-à-dire dans la saison de végétation. C'est en ce temps-là qu'on peut constater de grandes intumescences des eaux phréatiques. Les travaux de recherches ont confirmé que pendant la crue de 1954, par exemple, le territoire de Zitny ostrov a recu $201 \mathrm{~m}^{3} / \mathrm{s}$ d'eau au maximum, dont $63 \mathrm{~m}^{3} / \mathrm{s}$ sont apparus sur la surface. Cela signifie que les eaux d'infiltration passant par le sous-sol des levées atteignent sur certains secteurs 300 à $1400 \mathrm{l} / \mathrm{s}$. km, dont 0 à $980 \mathrm{l} / \mathrm{s}$. $\mathrm{km}$ peuvent surgir sur des surfaces protégées. L'affluence souterraine est la plus grande dans la région la plus élevée du cône de déjection (entre les kilomètres 1852 et 1810). La quantité maximale des eaux d'infiltration jaillit aux environs des kilomètres 1780 à 1770 .

La surface globale inondée par les eaux sauvages (dites intérieures) comportait en 1954 quelque 10000 ha. Le drainage des terrains environnants est par conséquent un problème sérieux, du point de vue hydraulique en même temps qu'agricole.

En aval de Komárno, l'influence du Danube sur les eaux phréatiques est sensiblement plus faible.

\section{Interventions du génie civil, à caractère historique.}

Les trouvailles de vestiges des âges de la pierre et du bronze faites aux alentours du secteur en question prouvent la présence dans ce territoire de colonies datant de l'âge préhistorique. Il est évident que la défense contre l'eau - cette force élémentaire de la nature - commença dés cette époque. Les premiers vestiges subsistant encore de la lutte de l'homme contre les inondations - ceintures de protection installées autour d'une colonie - proviennent déjà des temps historiques. Le premier document écrit enregistre la crue du Danube en l'année 1012, au cours de laquelle «bien des personnes et d'animaux ainsi que d'édifices ont été détruits ». L'ordonnance du roi Sigismond adressée en 1426 à la ville de Somorja est considérée comme Je début de la latte organisée contre les inondations; cette ordonnance décrétait la construction et la remise en état des levées de protection et des ceintures, devant être réalisées selon les instructions.

La construction systématique de levées de protection a commencé en 1850 sur la rive gauche et après 1890 sur la rive droite (fig. 14).

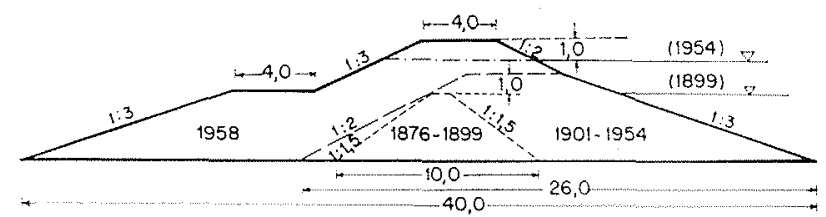

14/ Evolution du profil transversal des levées du Danube. Fortschreitende Entwicklung des Deichquerschnitts.

Certes, les levées ont assure une défense contre les inondations directes; leau a reapparu cependant derrière les levées, du faut dos précipitations, mais surtout comme ean fallissante. La lutte contre les eaux stagnanles o commencé en vue de la protection de lagriculturo la première association de drainage siesl fomoe sue hive gauche en 1854, done i polite quolques années après la construction systimulugu des lovées régulières de 
protection. L'évolution, dictée manifestement par l'intérêt direct de l'agriculture, est caractérisée par le fait que, dès 1876 , trois associations de ce genre fonctionnaient sur la rive gauche du Danube.

Outre la défense contre les inondations et le drainage des eaux nuisibles, les besoins de la navigation ne tardèrent pas à se présenter. Le fleuve, divisé en mille branches changeant bien souvent non seulement leur section, leur profondeur, mais aussi dans bien des cas leur lit, les bateaux ne pouvaient circuler qu'avec les plus grandes précautions. Les difficultés de la navigation peuvent être illustrées le mieux par deux données, selon lesquelles, d'une part les chòmages forcés dans la navigation se sont étendus en 1870 sur cent soixante-quatre jours et en 1785 sur cent seize jours, d'autre part qu'en 1877 quatre cent quarante péniches de plus étaient nécessaires pour alléger les sept cent quatre-vingts péniches demeurées en route. Sur les bancs de gravier et dans les divagations des lits, les glacons flottants se sont souvent arrêtés, des embâcles se formaient et les crues de débâcle débordaient souvent par-dessus les levées de protection relativement basses.

Par suite de cette grave situation, le désir de commencer les travaux de régularisation du fleuve se manifesta de plus en plus de divers côtés.

La protection des agglomérations humaines et des territoires agricoles contre les inondations et les eaux intérieures nuisibles, ainsi que le développement des transports par eau, ont imposé de façon impérjeuse, avec rythme et ordre d'importance divers, l'exécution et le développement de différents travaux.

Les ouvrages de défense contre les crues et de drainage, ainsi que de régularisation du lit, furent réalisés aux époques et avec l'importance résultant des besoins.

\section{Travaux de DÉFEnse contre les crues:}

Parmi les travaux de régularisation, c'est la construction des levées de défense contre les crues qui était très fortement influencée, peut-être même le plus, par les intérêts étroits locaux. D'une part, les innombrables bras du fleuve changeant continuellement leurs lits; d'autre part, les limites des domaines individuels déterminaient le tracé des levées de protection et, ni la nature du sous-sol, ni les principes de la régularisation des rivières n'étaient pris en considération. La largeur du champ d'inondation change capricieusement entre les valeurs extrêmes de 1,1 et $5,7 \mathrm{~km}$, et les levées traversent par-ci par-là de vieux bras remblayés par de la vase, endroits absolument mauvais au point de vue du sous-sol. Par conséquent, les levées ne remplissent pas, d'une part, leur ròle de régularisation du lit (ce qui ressort clairement de la fig. 2) et, d'autre part, elles ont d'innombrables points faibles, ce qui est prouvé par la plupart des crues qui ont eu lieu jusqu'à maintenant, ainsi que par les investigations géohydrologiques.

Après la construction des levées de protection, un nouveau problème s'est posé, à savoir que les crues, ne pouvant plus s'étaler sur de vastes territoires, déposent une partie de leur débit solide dans le champ d'inondation relativement étroit, par suite de quoi leur exhaussement a commencé. Suivant les mesures jusqu'ici effectuées, le rythme de l'exhaussement est de $1 \mathrm{~cm} /$ an sur le secteur du fleuve, du $\mathrm{km} 1850$ au $\mathrm{km}$ 1806. La cote ảes terrains pro-

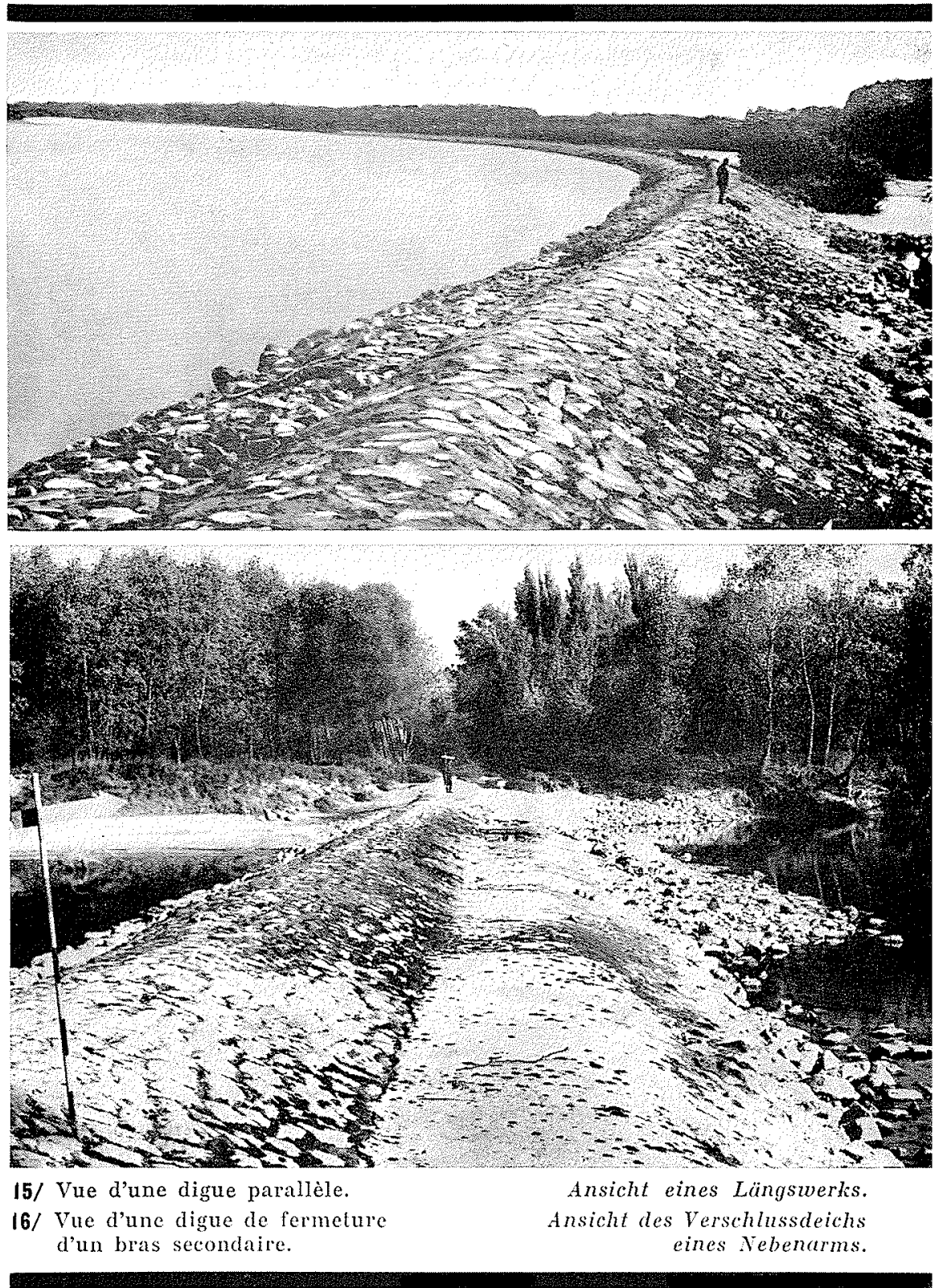

tégés restant invariable, l'exhaussement continuel du lit majeur a créé une situation de plus en plus grave.

\section{RÉgutarisation DU LIT :}

Les difficultés inhérentes à la navigation et la fréquence des crues de débâcle exigeaient d'urgence une intervention dans la vie du fleuve. La régularisation a eu lieu en deux étapes, d'abord pour les eaux moyennes, puis pour les basses eaux. Le projet de la régularisation aux eaux moyennes avec profil transversal constant du secteur BratislavaGönyü fut dressé en 1880-1881, dans le but d'assurer une profondeur de $2 \mathrm{~m}$ aumdessous du niveau des basses eaux ainsi que l'évacuation sans entraves des glaces. On a choisi comme niveau moven de base celui correspondant à la hauteur d'eau de $300 \mathrm{~cm}$ à l'échelle de Bratislava, avec un débit de $2886 \mathrm{~m}^{3} / \mathrm{s}$.

La régularisation des eaux moyennes a été effectuée entre 1886-1896. Les travaux de régularisation comprenaient en premier lieu, sur les deux rives, des ouvrages parallèles en moellons (fig. 15) construits jusqu'à la hauteur du niveau des eaux moyennes, des travaux de protection des berges, des coupures, et quelques digues transversales, même la fermeture de bras secondaires (fig. 16). 
La largeur de régularisation a été calculée à l'aide de diverses formules bien connues, puis modifiée sur la base de l'expérience. Ainsi donc, une largeur de régularisation de $300 \mathrm{~m}$ a été établie pour le secteur de grande pente, $325 \mathrm{~m}$ pour le secteur de transition et $380 \mathrm{~m}$ à l'aval du changement de pente, afin d'obliger ainsi l'eau, resserrée dans un lit uniforme, à atteindre la profondeur voulue. Le tracé du lit fut déterminé soigneusement à travers la large bande de divagations du fleuve - les principes de Fargue et de Girardon n'étant pas encor'e connus à cette époque - en longues droites et courbes douces, conformément à l'état des connaissances de l'époque en matière de régularisation de rivières, et en respectant rigoureusement l'économie. En créant de longs secteurs rectilignes, on voulait faciliter le passage de la glace. Au cours des travaux, $3600000 \mathrm{~m}^{3}$ de pierre brute furent mis en ruvre et $7800000 \mathrm{~m}^{3}$ de dragage ont été effectués.

Comme résultat de la régularisation, un lit principal constant s'est formé à la place de l'ancien Danube (voir fig. 2 et 4 ) et, de ce fait, les conditions de navigation aussi bien que celles de l'évacuation des glaces se sont considérablement améliorées. Toutefois, l'objectif original, d'assurer même à l'étiage un chenal de navigation d'une profondeur de $2 \mathrm{~m}$ au moins, proposé au début, n'était pas atteint.

Après l'achèvement de la régularisation aux eaux moyennes, les insuffisances de cette régularisation ne tardèrent pas à se présenter, notamment:

a) Sous l'effet du resserrement du lit, le niveau des eaux moyennes s'est relevé au début - entre 1887 et 1894 - à l'extrémité supérieure du secteur, d'environ $80-90 \mathrm{~cm}$ à Bratislava. Cette élévation du niveau n'a diminué progressivement qu'après 1900. Sous l'effet de l'élévation du niveau d'eau, l'exhaussement du fond du lit, du fait de la diminution de la pente, était considérable, en premier lieu dans le secteur Devin-Bratislava (du km 1880 au $\mathrm{km} \mathrm{1869)}$; done les conditions de la navigation empiraient. Dans le secteur aval de l'exhaus-

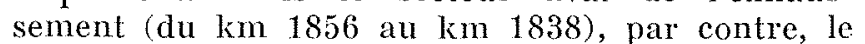
lit s'est mis à s'approfondir à la suite de l'accroissement de la pente, pendant qu'il a commencé à se combler intensément, sur le secteur de transition, au point du changement de pente - où les hautes eaux et les eaux moyennes peuvent s'étaler librement.

b) Le lit moyen, se composant des secteurs droits limités par des digues parallèles, s'est révélé trop large pour les eaux basses. Par conséquent, d'une part, le chenal de navigation a continuellement changé de place; d'autre part, le dépôt du débit solide concentré au lit moyen a augmenté, la migration des banes de gravier a commencé, de mauvais passages et des étranglements de la voie de navigation se sont formés.

c) L'eau, après avoir déposé le débit solide dans le lit principal, pourrait librement passer à travers les ouvertures ménagées dans les digues parallèles, dans les bras secondaires. Donc, les ouvertures ont causé à l'étiage une perte d'eau inutile pour le lit principal, d'où une diminution du pouvoir érosif et de la profondeur.

d) Notons encore que les fermetures secondaires projetées dans les bras n'ont pas été réalisées, surtout par raison d'économie, alors que si elles avaient été construites en nombre adéquat à une hauteur convenable, elles auraient facilité le remblayage des bras secondaires et diminué l'exhaussement du lit principal.

Les exigences sans cesse croissantes de la navigation réclamaient d'urgence de commencer la régularisation aux basses eaux. La régularisation aux eaux moyennes effectuée, l'application de la méthode du resserrement avec ouvrages parallèles ne se révélait pas, malgré ses défauts, vaine et inutile. Elle a permis de réaliser rapidement sur ce secteur la régularisation aux basses eaux sur la base du principe de régularisation à «profil normal variable $»$ exposé par H. Girardon en 1894, à l'occasion du VI' Congrès International de Navigation, à La Haye. L'idée de la régularisation aux basses eaux commencée au tournant du siècle était d'établir d'une manière convenable le tracé du thalweg en tenant compte des formes naturelles du lit, de sa profondeur, de sa pente, de ses changements de vilesse, et de fixer cette ligne de façon durable à l'aide d'ouvrages de régularisation. En mème temps, les pertes d'eau du lit principal devaient ètre réduites de façon que son débit soit, mème à l'étiage, suffisant pour la navigation. Pour donner un tracé convenable au chenal de navigation et pour le fixer, on a construit des épis (voir fig. 4). Pour réduire les pertes d'eau du lit principal, une partie des onvertures laissées dans les digues parallèles a été fermée, et des séries de fermetures secondaires ont été créées dans les bras. Finalement, comme moyen supplémentaire, le dragage fut employé.

Le caractère spécial de la régularisation résidait dans le fait que le chenal d'étiage correspondant aux principes de Fargue et de Girardon devait être développé à l'intérieur des digues parallèles de tracé défini. D’autres difficultés résultaient de ce que les embouchures des bras secondaires d'une certaine importance devaient également être prises en considération en établissant le tracé du thalweg.

Sous l'effet de la régularisation aux basses eaux, le lit s'est développé avantageusement au point de vue de la navigation, de facon provisoire, et les conditions de la navigation se sont beaucoup améliorées.

Inutile de dire que cette régularisation ne pouvait naturellement assurer le développement de l'état d'équilibre du secteur considéré. A cette époque, on ne connaissait pas encore les facteurs sur la base desquels on aurait pu calculer les corrélations entre le débit d'eau et le charriage, à l'aide desquelles le bilan hydrologique du débif solide du Haut-I anube et les conditions du développement du lit en équilibre auraient pu être déterminées.

Après que la première guerre mondiale eut éclaté, la cadence des travaux de régularisation se ralentit et se limita jusqu’à 1927 - époque de la création d'une Commission Technique Commune hongroise ef tchécoslovaque - au strict minimum nécessaire à l'entretien. Pendant l'interruption forcée des travaux, les insuffisances du système de régularisation suivi jusque-là se sont montrées; on en a tenu compte en reprenant les travaux de régularisation au niveau de l'étiage navigable déterminé par la Commission Internationale du Danube en $1932(1)$.

(1) Niveau atteint ou dépassé, en moyenne, pendant 290 jours de la période de navigation de 300 jours comptée du 10 mars au 25 décembre. 


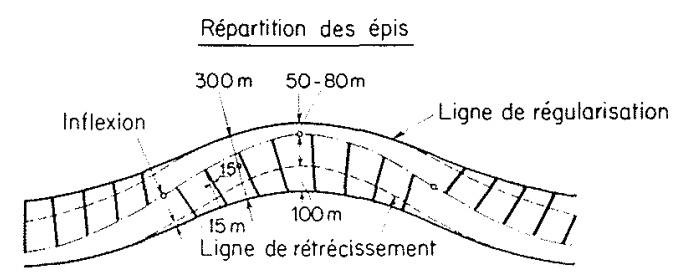

Profil en long

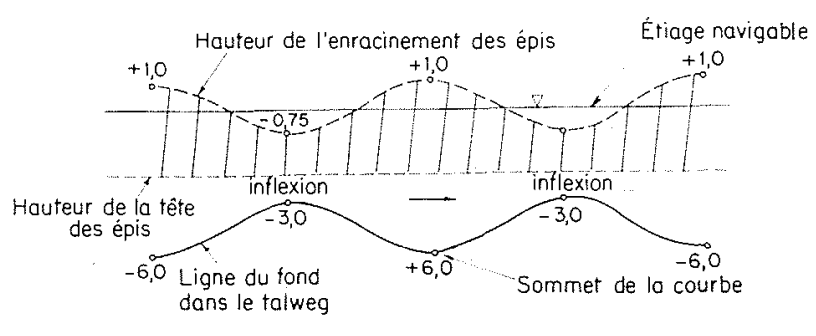

Profil transversal dans I'inflexion
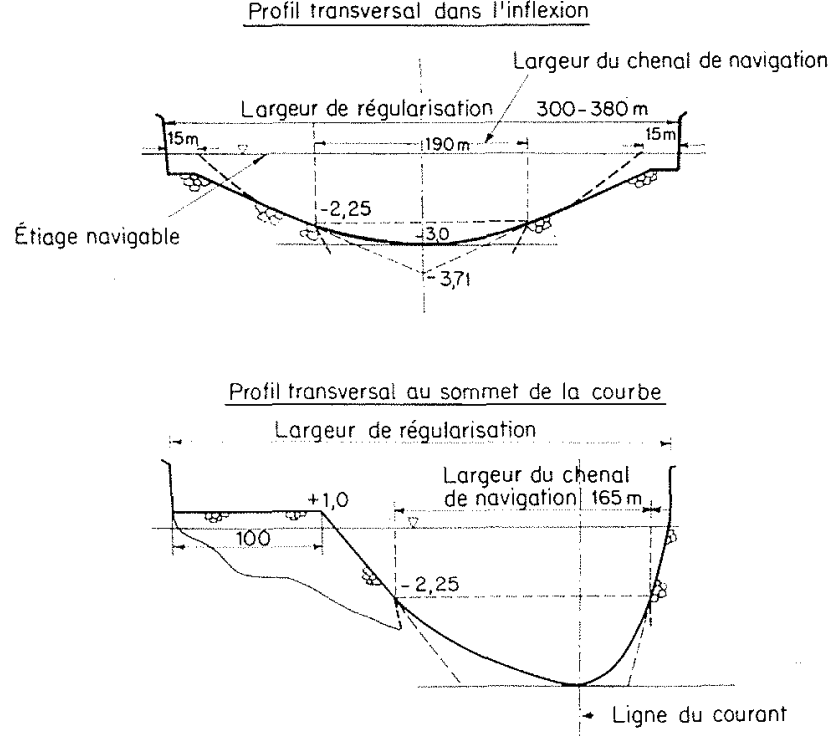

17/ Schéma de principe du systène modifié de régularisation aux basses eaux.

Prinzipschema des veränderten Systems der Niederwasser-Regulierung.

a) Les observations ayant montré que la proPondeur d'eau n'est pas constante aux divers points des courbes, mais augmente à partir du point d'inflexion jusqu'au sommet, on envisagea de fixer la profondeur au-dessous de l'étiage navigable à $6 \mathrm{~m}$ aux sommets des courbes et à $3 \mathrm{~m}$ aux points d'inflexion (fig. 17), en supposant qu'elle varie entre les deux valeurs extrêmes suivant une sinusoïde.

b) Selon les observations, la largeur du lit mineur n'est pas non plus constante. Aux inflexions, le profil transversal est symétrique, de forme parabolique, et la largeur de régularisation de $300 \mathrm{~m}$ est un peu trop grande; tandis qu'au sommet les bancs de gravier déposés sur le côté convexe du lit moyen engendrent un rétrécissement allant jusqu’à $100 \mathrm{~m}$. Par conséquent, il fallait procéder au rétrécissement du lit. Celui-ci atteint $100 \mathrm{~m}$ (c'esta-dire $33 \%$ ) aux sommets des courbes et $30 \mathrm{~m}$, soit $10 \%$, aux points d'inflexion; il est uniformément réparti sur les deux côtés. c) Il paraissait utile de dresser le plan du chenal de navigation. La largeur du chenal fut fixée à $150 \mathrm{~m}$ et sa profondeur à $2,25 \mathrm{~m}$. Les épis projetés - dont la hauteur et l'inclinaison augmentent graduellement à partir du point d'inflexion vers les sommets - ne seront construits que jusqu'au bord du chenal projeté.

Du point de vue de l'amélioration des seuils et de l'entretien du chenal de navigation, le système de régularisation modifié s'est montré efficace.

Le résultat peut être illustré par deux exemples.

Tandis que sur le secteur du km 1850 au $\mathrm{km} 1794$. la navigation devait etre interrompue en moyenne pendant quatre-vingt-quatre jours durant la période libre de glace au cours de la décennie de 1867 1876 , elle ne fut pas arrêtée un seul jour, même en 1947, année aux eaux extrêmement basses.

L'effet de la régularisation est encore mieux illustré par un exposé de M. Horváth dans un rapport sur l'entretien du chenal de navigation dans le secteur du Danube entre Rajka et Gönyü (km 1850-1791), présenté au XXo Congrès International de Navigation (Baltimore, 1961). M. Horváth a introduit, pour caractériser l'état de la voie navigable, l'indice d'insuffisance effective de mouillage par rapport à la profondeur de $25 \mathrm{dm}$ voulue, cet indice tenant compte également de la durée d'étiage pendant laquelle le passage difficile se présente. L'indice ainsi déterminé se chiffrait, entre 1921. 1944, par $1066 \mathrm{dm} . j o u r$, tandis qu'entre 1946 et 1954, il n'était que de $854 \mathrm{dm}$.jour. Les débits moyens dans les deux époques considérées étaient respectivement de 2200 et $1960 \mathrm{~m}^{3} / \mathrm{s}$, et la deuxième époque comprenait l'année 1947, extraordinairement pauvre en eau $\left(Q_{\text {moven }}=1390 \mathrm{~m}^{3} / \mathrm{s}\right)$.

Tous ces résultats n'ont amélioré que les conditions de navigation; et le problème le plus difficile du secteur considéré, l'exhaussement du lit, n'a pas été non plus résolu par cette méthode.

\section{L'aménagement du Danube depuis l'année 1945.}

La deuxième guerre mondiale a aussi marqué de son empreinte le bassin du Danube. Les événements militaires directs ont beaucoup limité el rendu difficile la navigation, étant donné que le fleuve était miné et que les navires avariés formaient des obstacles sérieux pour la navigation.

L'apparition périodique de mauvais passages, surtout dans les kilomètres $1804,1805,1806,1807$, $1810,1811,1827,1829,1830,5,1831,1832,1833$, 1837 , 1838, témoignait du fait que, pendant la guerre, l'entretien des ouvrages de régularisation fut forcément négligé. Il en résultait des brèches daus les dignes parallèles et, par suite, des pertes considérables d'eau du lit principal. Des dépôts locaux et des banes de gravier se sont formés dans le lit. L'eau des bras secondaires, rentrant dans le lit principal et provoquant des remous locaux, a amené en amont du confluent une diminution rapide de la force tractrice et un processus de sédimentation dans quelques secteurs exposés. Dans ces conditions, les épis endommagés ne pouvaient, eux non plus, satisfaire à leur tâche. Cette situation entrainant des conséquences économiques très sérieuses, les experts hongrois et tchécoslovacues ont décidé de prendre des mesures concrètes. 


\section{J. FURDICK ET K. STELCZER}

Ces mesures indispensables peuvent être caractérisées de la facon suivante :

- Reconstruction des ouvrages de régularisation aux basses eaux et leur achèvement;

-- Reconstruction des ouvrages de régularisation aux eaux moyennes dans certains secteurs;

- Dragage général du lit el dragage des seuils;

- Dépòt des matériaux de dragage dans les bras secondaires;

- Correction du tracé du lit principal à quelques endroits;

- Correction du tracé des levées; el construction d'épis dans le champ d'inondation.

Les travaux, qui avaient au début un caractère de reconstruction, finirent par avoir le caractère d'une activité de correction consciente de ses buts et systématique. Cette activité devait être dirigée et réglementée, et c'est pour cette raison que les gouvernements hongrois et tchécoslovaque ont renouvelé l'activité de la Commission Technique Commune hongroise et tchécoslovaque, qui est toujours en fonction.

La Commission a discuté les différentes études théoriques et expérimentales qui ont servi de base à la préparation du nouveau «Projet global de régularisation du secteur à passages difficiles du Danube hongrois-tchécoslovaque» terminé en 1950.

Dans ce projet, les experts des deux pays ont démontré la nécessité d'un aménagement planifié du secteur commun du Danube, du point de vue économique et technique, en liaison étroite avec les divers travaux de régularisation effectués au cours des années de 1898 à 1950 .

Ce projet global devrait être pris pour base des autres projets ultérieurs plus détaillés. Etant donné cependant que l'on compte exploiter l'énergie hydraulique de ce secteur, on ne pouvait pas développer et réaliser les propositions originales dans toute leur étendue, et par là on a réduit à la mesure indispensable les travaux exécutés dans le lit moyen et dans le chenal d'étiage.

Leur importance ressort du tableau $V$.

Les niveaux d'étiage observés au cours des années 1951 et 1952 , puis les expériences acquises pendant la crue de 1954 et les études effectuées dans cette période ont cependant reporté de nouveau l'attention vers l'exhaussement du lit. Vu que le problème a acquis une importance croissante, il a imposé un changement de conception de la régularisation. Dès le début des travaux de régularisation, on a formulé l'exigence essentielle d'assurer une navigation permanente et sans interruption. Cette exigence a soulevé, entre autres problèmes urgents, avant tout celui de trouver le moyen d'assurer une stabilité permanente au profil en long.

On a constaté de nouveau que les causes du dépôt de sédiments étaient les suivantes :

a) le changement brusque de la pente à Palkovičovo (km 1810), comme suite auquel la force tractrice diminue subitement;

b) dans le secteur de Rajka-Palkovičovo (km 18501810), le lit majeur est trop large. Il en résulte une sédimentation accrue des suspensions dans la période de crues, et un exhaussement permanent du terrain inondé;

c) les vastes forêts situées dans le champ d'inondation contribuent aussi à la diminution de la vitesse de l'eau, favorisant ainsi la sédimentation; d) pour assurer la navigation sans interruption, on a été obligé d'augmenter la hauteur des digues parallèles ainsi que des fermetures des bras secondaires, suivant l'exhaussement du fond du lit. En conséquence, la masse des matériaux solides passant dans les bras a diminué; la concentration des eaux moyennes dans le lit principal n'était cependant pas assez forte pour augmenter suffisamment la force tractrice et favoriser le morcellement du débit solide.

Dans ces conditions, on a supposé avec raison que, dans quelques décennies, le Danube aura son lit surélevé.

Ces circonstances ont en, par l'infiltration intensifiée des eaux, une influence néfaste sur les terrains environnants, terrains importants du point de vue agricole. On a proposé par la suite:

$1^{\circ}$ d'enlever, par dragage, approximativement 1 million de $\mathrm{m}^{3} / \mathrm{an}$ du matériau solide du lit entre les km 1837 el 1810 ;

$2^{\circ}$ de couper le sous-bois au voisinage des rives du lit principal et de renforeer ainsi la force tractrice de la crue;

3" d'efiectuer les études complexes relatives au mouvement et à la répartition des matériaux solides dans la section transversale;

$4^{\circ}$ de chercher les moyens d'utiliser les matériaux du dragage pour les travaux de régularisation ainsi que pour d'autres buts;

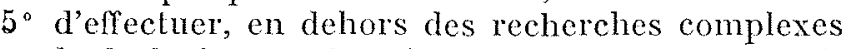
hydrologiques, des études sur modèle réduit (fig. 18) dans les conditions suivantes:

a) les quantités de matériaux dragués, pendant cinq années, atteindront 5 millions de $\mathrm{m}^{3}$,

b) des digues d'épis seront construits dans le champ d'inondation,

c) le sous-bois sera coupé, rendant libre une largeur de $300 \mathrm{~m}$ pour l'écoulement des crues,

d) certains bras secondaires seront liquidés.

Depuis 1954, l'activité de régularisation a augmenté et les mesures énumérées ont été en partie réalisées. Le tableau VI en donne les caractéristiques.

Parallèlement aux travaux mentionnés, le projel de créer un lit uniforme et stable a pris forme. Des études considérables doivent précéder son éta blissement. Les problèmes suivants restaient toujours à résoudre:

- la régularisation du lit principal;

- l'amélioration des passages difficiles;

- la suppression de l'écoulement dans les bras secondaires;

-. l'aménagement du champ d'inondation;

- la collecte ininterrompue des données d'observation et la continuation des recherches.

\section{Les perspectives} des travaux de régularisation.

Dans la suite, on envisage de réaliser la formation d'un lit principal unifié.

Celui-ci mettrait en harmonie l'écoulement des crues et des eaux moyennes, et assurerait l'évacuation sans entraves des glaces. Les interventions techniques en vue de cette réalisation doivent être projetées de façon qu'elles soient en harmonie avec les ouvrages de régularisation déjà construits. On doit avoir soin de poursuivre, simullanément avec les travaux de régularisation du lit majeur, ceux de régularisation aux basses eaux. 


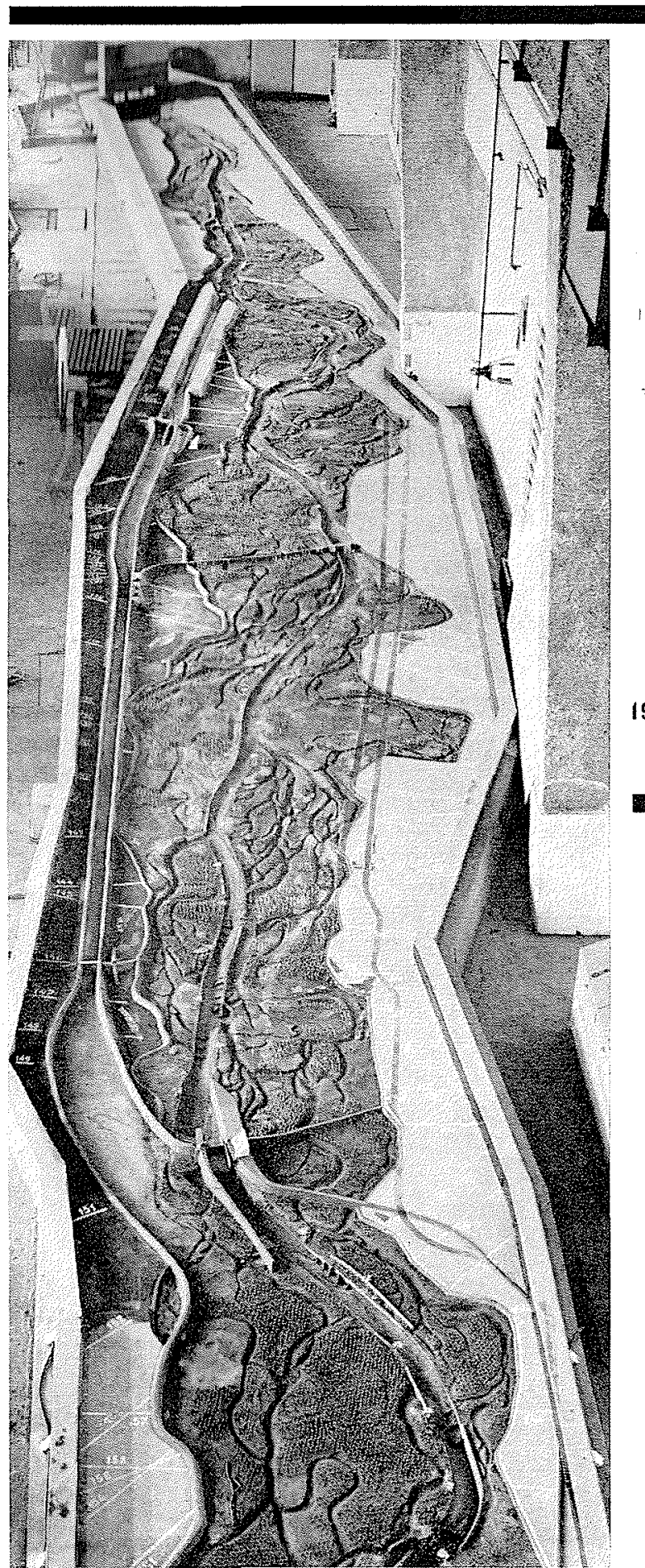

18/ Modèle à l'échelle réduite d'un secteur du Haut-Danube au horatoire d'hydratique de I'Institut de Recherches des Ressources Hydruliques à Budapest. Modell eines Abschnits der Oberen Donau in der Versuchsanstalt für Hydraulik

dos Forschungsinstituts für Wasserwirtschaft Budapest.

a) Dans le lit moven, ce sont les interventions suivanles qui aideraient à ménager les conditions de l'écoulement :

La tache primordiale est d'égaliser les débits le long du profil en long, d'empêcher les pertes d'eau affectant le lit au droit de la sortie des bras. Ceci pouvait se faire en premier lieu en fermant progressivement les systèmes de bras.

Cetle exclusion progressive des bras secondaires du transport de l'eau faciliterait beaucoup l'amélioration des conditions de pentes. Il faut cependant souligner le fait qu'en formant la ligne unifiée de la rive et en fermant les bras secondaires, l'amé- nagement des conditions de pentes les plus avantageuses ne se réalise pas encore. Dans cette question, un rôle important est dévolu aux crues dépassant $4000 \mathrm{~m}^{3} / \mathrm{s}$ étalées jusqu'ici, car il faut assurer également leur écoulement sans qu'elles occasionnent des relèvements nuisibles du plan d'eau. Ainsi l'on peut arriver à prévenir le dépôt nuisible du débit solide en suspension. Un aménagement favorable des conditions de pentes dépend pour beaucoup d'un rythme adéquat des travaux d'intervention, ce qui nécessite l'étude continue du problème.

Le succès de l'aménagement des conditions d'écoulement et des pentes influencerait fort celles du transport du débit charrié dans le secteur.

La part prépondérante du charriage s'effectue dans le domaine des eaux moyennes. Près de $70 \%$ de la matière charriée annuellement est transporté par les débits de $1000-3400 \mathrm{~m}^{3} / \mathrm{s}$ correspondant aux niveaux de 190-530 $\mathrm{cm}$ à l'échelle de Bratislava. Donc, le transport de matériaux par des débits inférieurs à $3400 \mathrm{~m}^{3} / \mathrm{s}$ est à celui des débits supérieurs à cette quantité à peu près dans le rapport de deux à un.

Dans la situation présente, la capacité de transport du lit principal diminue du fait des pertes d'eau se produisant vers les bras secondaires, et une partie de la matière transportée se dépose dans le lit principal. En amont des points de réembouchure des bras, les vitesses diminuent du fait du remous dù à l'accroissement du débit, et des dépôts de matières transportées se produisent.

L'effet des bras secondaires esquissé dans ce qui précède peut être évité en supprimant les systèmes de bras, mais, ce faisant, nous rendrons seulement plus uniforme la répartition de la matière se dépo. sant sur le secteur et retarderons ainsi considérablement l'exhaussement de certaines parties du secteur. On voit que ce mode d'intervention n'arrête point à lui seul le processus d'exhaussement du secteur, car le transport du débit solide du sectem. tout entier est déterminé par la capacité de transport des profils de Bratislava et de Medvedov. 
b) Les interventions visant la formation favorable du lit des eaux moyennes entrainent et rendent nécessaire la formation - en harmonie avec les conditions nouvelles du lit moyen - du lit majeur uniforme. Sans la régtilarisation de l'écoulement des crues, il n'est guère possible d'entretenir le lit des eaux moyennes et le champ d'inondation.

On se propose de réaliser le tracé favorable du lit majeur en premier lieu, avec une végétation adéquate des champs d'inondation, par des «digues vivantes ». Celles-ci poursuivent deux buts. En premier lieu, elles assureraient le tracé miforme des champs d'inondation; par ailleurs, les raccordements transversaux s'embouchant à elles neutraliseraient les parties se trouvant à l'ombre au point de vue de l'écoulement et favoriseraient leur remblayage.

La concentration des débits de crue doit s'effectuer progressivement et avec circonspection, car sans cela nous pourrions influencer d'une facon dangereuse les niveaux des crues.

Toutefois, la formation favorable et définitive de nouvelles conditions d'écoulement ne peut ètre espérée qu'après l'achèvement des deux étapes de travaux exposés ci-dessus et exécutés en pleine harmonie.

Les opinions des experts s'accordent sur les principes fondamentaux de l'aménagement général, mais les idées concernant les interventions pratiques sont encore divergentes jusqu'à présent.

Un groupe d'experts affirme que l'effet des interventions projetées sur le secteur du Danube entre Rajka et Medvedov ( $k m$ 1850-1805) sur les caractéristiques géométriques du tracé n'est pas tellement considérable qu'il puisse influencer sur le rôle du processus géologique, cause principale de l'exhaussement et de la rupture de pente à Palkovičovo. Ils estiment que d'autres interventions sont encore nécessaires pour résoudre la question et citent en premier lieu le rétrécissement du lit à l'aval de la rupture de pente. De telles interventions aideraient à faire avancer le débit solide, et ainsi il deviendrait possible de supprimer sur le secteur en question les travaux de dragage, et limiter ceux-ci exclusivement à l'entretien du chenal de navigation aux basses eaux. Ils motivent cette façon de procéder par le caractère double du Danube supérieur. Ils voudraient réaliser l'équilibre du secteur divisé par des systèmes de bras à l'aide des procédés exposés.

Sur le secteur à l'aval de la rupture de pente, à

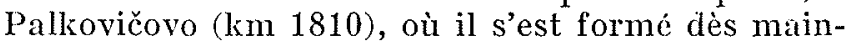
tenant un large lit d'eaux moyennes plus ou moins unifié - un champ d'inondation exempt de branches - on se propose de donner par des remblais de gravier et des ouvrages parallèles un alignement uniforme aux rives d'une hauteur correspondant au débit d'environ $3000 \mathrm{~m}^{3} / \mathrm{s}$. C'est en adoptant les dimensious géométriques et la forme les plus avantageuses pour le lit, que l'on croit pouvoir mettre en mouvement progressivement le débit solide se déposant sur ce secteur et le transporter plus loin à un point où le drogage des dépôts concentrés peut se faire dans des conditions plus avantageuses.

L'autre groupe d'experts estime que la tâche peut être résolue en aménageant de façon adćquate les rives concaves et ell concentrant le débit par une conformation judicieuse des rives convexes, et n'attribue guère d'importance aux conséquences hydrauliques, géologiques et morphologiques de la rupture de pente à Palkovičovo. Ce groupe croit que le rétrécissement du lit des eaux moyennes est réalisable par la voie naturelle en corrigeant le tracé et en aménageant les rives concaves, et en partie convexes, entrant en ligne de compte. Ils supposent qu'à la suite des interventions ainsi exécutées, les travaux de dragage peuvent ètre réduits au minimum. Pour l'avenir, on tient compte du fait qu'à la suite de l'aménagement des chutes du secteur autrichien, le débit solide du Danube diminue progressivement.

Les deux conceptions tendent à modifier entièrement la force tractrice du fleuve. Toutefois, la solution de cette question exige de poursuive les observations, les recherches et les travaux en laboratoire.

Nous avons montré que la question la plus délicate de la régularisation du secteur, à savoir la méthode de modification de la force tractrice, est inconnue à ce jour. La divergence des opinions s'explique par le fait que le secteur en question est, du point de vue de la régularisation, le plus délicat après celui des Portes de Fer.

Les interventions régulatrices jusqu'ici effectuées - et qui se sont appuyées sur des travaux de calcul et d'observation très approfondis - ne pouvaient pas résoudre le problème. Ceci s'explique en premier lieu par le fail que les événements de la première guerre mondiale ont empêché la continuation, et l'achèvement des travaux de régularisation de grande envergure exécutés au tournant du siècle. Dans la période subséquente, jusqu'à la fin de la seconde guerre mondialc, Ja situation économique ne permettait pas l'étude complexe des problèmes de régularisation du secteur, et encore moins l'exécution des travaux de grande envergure dont ces études auraient démontré la nécessité. Les travaux exécutés à cette époque servaient en premier lieu à assurer la profondeur de navigation voulue, ce en quoi ils ont d'ailleurs relativement bien réussi.

Actuellement, les phénomènes exposés dans ce qui précède imposent cependant d'effectuer avec circonspection des interventions bien étudiées.

Les travaux et études préparant cette intervention de grande envergure, dont les experts s'occupent depuis plusieurs années et dont nous venons d'exposer les buts, peuvent fournir une base solide à la solution des tâches complexes de la régularisation.

Il faut remercier les experts des travaux hydrauliques des deux Etats riverains d'avoir effectué durant cie longues années, de commun accord, leurs observations et recherches.

Par ces travaux, ils ont fourni des données de grande valeur pour effectuer des interventions en vue de modifier les processus naturels. Nous vondrions espérer que les résultats de ce travail contribueront à élargir et enrichir les comnaissances théoriques et pratiques de la régularisation des rivières.

\section{Bibliographie.}

S. Fekete. - Die Regulierung der Ungarischen Oberen Donau. Budapest, 1899.

E. de Kyassay. - Le Danube international. Budapest, 1916. 
\%. Kanowy. - Résultats provisoires des études relatives au charriage de débit solide du Haut-Danube (en hongrois, aveo l'sumes en allemand et en russe). Vizügyi Közlemengek (Revue d'Hydraulique), Budapest, no $1,1951$.

K. Tönx. - Le Danube et sa régularisation (en hongrois). Edition de l'Académie des Sciences, Budapest, 1952.

S. Honvath, - La voie navigable du Danube et la Hongrie (en hongrois, avec résumés français, russe et allemand). Vizügyi Közlemények (Revue d'Hydraulique), Budapest, $n^{\circ} 4,1954$.

K. 'Tönx. - Les conditions des glaces et des seuils des yoies navigables de Hongrie (en hongrois, avec résumés français, russe et allemand). Vizügyi Közlemények (Revue d'Iydraulique), Budapest, no $3,1956$.

O. Ruzrcka. Etude sur les travaux de régularisation du Danube (en tchèque). Edition SVII, Bratislava, 1956.
I. ZiLincan. - Etude sur le secteur de passages difficiles du Danube (en slovaque). Bratislava, 1958.

S. Honvath. - Maintenance of navigation channel over the shallow stretch of the Danube river between the villages Rajka and Gönyü (avee résumé français). XXth International Navigation Congress, section 1 , question 5 . Baltimore, 1961.

J. Szolgay. - Nouvel aspect du rehaussement de la partie supéricure du secteur hongrois-tchécoslovaque du Danube eu égard aux recherches relatives au débit solide charrié et en suspension (en slovaque). VUV, Bratislava, 1962.

J. Csoma et J. Szmagr. - Résumé et critique des études relatives a la régularisation du Haut-Danube (manuserit en hongrois). Vituki, 1962.

\section{Zusammenfassung}

\section{Die Probleme der Materialablagerung im Bette der Donau zwischen Devin und Szob}

\section{Von J. Furdick* und K. Stelczer * *}

Durch das Tor von Dévin in den Karpethenbecken tretend teilt sich die Donau auf eine für die Mündungsstrecke kennzeichnende Weise in viele Arme und bildet an der etwa $60 \mathrm{~km}$ langen Strecke zwischen Bratislava und Gônyü eine wahrhaftige Inselwelt. Infolge der Verbreitung und des geologisch bedingten Gefällebruches legt sie hier den grössten Teil ihres geschleppten Geschiebes ab. Die Verbreitung des Flusses und der grosse Geschiebeanfall war immer, und ist noch heute ein grosses Problem für die Ingenieure.

In der Mitte des vorigen Jahrhunderts wurde zuerst die Verbreitung des Hochwassers durch Bau von Hochwasserschutzdämmen aufgehoben. Später, in den Jahren von 1886 bis 1896 wurde zwecks Sicherung eines der Schiffahrt geeigneten einheitlichen Flussbetes durch Errichtung von Bruchsteinparallelwerken die Mittelwasserregulierung mit ständigen Querschnitten durchgeführt.

Dank der Hochwasserschutzdämmen konnten mächtige Gebiete vom Hochwasser geschützt werden, da aber die Hochwasser sich nunmehr nicht unbehindert verbreiten konnten, legten sie das Geschiebe in dem verhältnismässig engen Vorland ab. Die Verlandung erreichte $1-2 \mathrm{~cm}$ pro Jahr, und rasch bildete sich ein Hangbett aus.

Infolge der Mittelwasserregulierung bildete sich ein stündiges Hauptbett aus, so dass die Verhältnisse der Schiffahrt wie auch die der Eisabfünrung verbessert wurden, doch konnte man die Betterhöhung und die damit verbundene ständige Umtiefewanderung nicht verhindern.

Die stets zunehmende Ansprïche der Schiffahrt drängten zur Niederwasserregulierung. Die zur Zeit der Jahrhundertwende begonnene Niederwasserregulierung wurde auf Grund des im Jahr 1894 von Girardon an dem Navigationskongress in Haag bekanntgegebenen Prinzips "Regulierung mit änderlichem Normalprofil " durch Errichtung von Sporenbauten durchgeführt.

Mit der Niederwasserregulierung verbesserten sich zwar wesentlich die Navigationsverhältnisse, jedoch die technischen Eingriffe konnten das Problem der Betterhöhung nicht endgültig lösen. Die Lösung des Problems des sich ständig erhöhenden Dunaubettes und Vorlandes wartet noch auf die Ingenieure unserer Tage. Zur raschen und endgültigen Lösung des Problems drängt die Tatsache, dass auf der verlandenden Strecke infolge der verhältnismässig immer niedriger werdenen Hochwasserschutzdämmen die Hochwassersicherheit immer kleiner wird, die landwirtschaftlich benützten Gebiete des Ueberschwemmungsgebietes sich immer mehr versumpfen und die Kosten ihrer Entwässerung immer mehr betragen.

Im Interesse der Lösung des Problems wurde in den vergangenen Jahrzehnten eine ganze Reihe von Serienmessungen an Ort und Stelle durchgeführt, um die hydrologischen Kennwerte um so tiefer aufschliessen zu können. Es wurde auch das Kleinmodell der interessierten Strecke im Massstabe 1:2 000 horizontal und 1:150 vertikal verfertigt. Im Sinne des von dem Ungarisch-Tschechoslowakischen Gemeinsamen Technischen Komitee angenommenen Regulierungsprinzip wurde mit Berücksichtigung der bisherigen Eingriffen und deren Wirkung die Ausbildung eines einheitlichen Hauptbettes zum Ziel gesteckt. Mit der Ausbildung eines einheitlichen Flussbettes soll die weitere Verbreitung des Hochwassers verhindert, die Schleppkraft des Flusses vergrössert und damit die Möglichkeit einer weiteren ungünstigen Ablagerung behoben werden. Das Geschiebe muss sich dann auf einem geeigneten Orty wie z.B. bei einer zu bauenden Betonfabrik/konzentriert ablagern.

Solange die Flussregulierung durchgeführt wird, soll die weitere Betterhöhung mit Baggerung von jährlich $1000000 \mathrm{~m}^{3}$ Kiesel eingeschränkt werden.

* Ing., Director des Forchungsinstituts für Wasserwirtschaft, Bratislava (Tschechoslowakei).

** Direktor der Forschungsanstalt für Wasserwirtschaft, Budapest (Ungarn). 
TABLEAUX

I. - Le débil du Danube dans les différents profils en pour cent du débit passant par Bratislava

\begin{tabular}{|c|c|c|c|c|c|}
\hline \multirow{3}{*}{$\begin{array}{c}\text { SECTTON } \\
\text { DE } \\
\text { JAUGEAGE } \\
\\
(\mathrm{km}) \\
\end{array}$} & \multicolumn{5}{|c|}{ Date dU JaUgEage ET DÉBT TOTAL a Bratislava } \\
\hline & $17-1-1961$ & $21-9-1960$ & $\begin{array}{c}20 / 22-6 \\
1961\end{array}$ & $\begin{array}{c}16 / 17-5 \\
.1961\end{array}$ & $\begin{array}{c}13 / 14-7 \\
1955\end{array}$ \\
\hline & $1005 \mathrm{~m}^{3} / \mathrm{s}$ & $1958 \mathrm{~m}^{3} / \mathrm{s}$ & $2998 \mathrm{~m}^{3} / \mathrm{s}$ & $4002 \mathrm{~m}^{3} / \mathrm{s}$ & $6702 \mathrm{~m}^{3} / \mathrm{s}$ \\
\hline 1869,04 & 100 & 100 & 100 & 100 & 100 \\
\hline 1860,00 & 92 & 89 & 92 & 92 & 65 \\
\hline 1851,63 & 79 & 81 & 88 & 89 & 87 \\
\hline 1847,85 & 84 & 95 & 85 & 70 & 58 \\
\hline 1842,40 & 99 & 97 & 83 & 68 & 50 \\
\hline 1833,10 & 76 & 74 & 62 & 65 & 48 \\
\hline 1825,00 & 88 & 96 & 78 & 67 & 53 \\
\hline 1821,07 & 65 & 82 & 66 & 51 & 40 \\
\hline 1816,85 & 84 & 84 & 76 & 64 & 50 \\
\hline 1810,40 & 99 & 85 & 68 & 63 & 43 \\
\hline 1806,00 & 99 & 99 & 89 & 92 & 73 \\
\hline 1802,37 & 99 & 99 & 90 & 90 & 75 \\
\hline
\end{tabular}

11. - Débits caractéristiques du secteur hongrois-tchécoslovaque du Danube

\begin{tabular}{|c|c|c|c|c|c|}
\hline \multirow[b]{2}{*}{ Profil } & \multirow[b]{2}{*}{$\mathrm{km}$} & \multirow{2}{*}{$\begin{array}{c}\text { SUPER- } \\
\text { FICIE } \\
\text { DU B.V. } \\
\text { en } \\
1000 \mathrm{~km}^{2}\end{array}$} & \multicolumn{3}{|c|}{ DÉnirs $\left(\mathrm{m}^{3} / \mathrm{s}\right)$} \\
\hline & & & $\underset{\text { mini- }}{\text { Mal }}$ & Moyen & $\begin{array}{l}\text { Maxi- } \\
\text { mal }\end{array}$ \\
\hline Bratislava. & 1870 & 131 & 570 & 2025 & 10900 \\
\hline Dunaremete. & 1826 & 131 & 570 & 2025 & 9600 \\
\hline Gönyü. . . . . & 1791 & 150 & 583 & 2105 & 8800 \\
\hline Komárno-Komárom. & 1768 & 170 & 605 & 2295 & 8900 \\
\hline Confluent du Hron. . & 1716 & 173 & 613 & 2350 & 8700 \\
\hline Confluent de l'Ipel-Ipoly. . & 1708 & 178 & 615 & 2375 & 8900 \\
\hline
\end{tabular}

11.. - Vitesse moyenne dans le lit principal, en $\mathrm{m} / \mathrm{s}$

\begin{tabular}{|c|c|c|c|}
\hline \multirow{3}{*}{$\begin{array}{c}\text { DÉBIT } \\
\text { A } \\
\text { BratisLaA } \\
\left(\mathrm{m}^{3} / \mathrm{s}\right)\end{array}$} & \multicolumn{3}{|c|}{ SEction de L'Écheile de } \\
\hline & Baika & Dunaremete & Medvadoy \\
\hline & $\mathrm{km} 1848$ & $\mathrm{Jm} 1826$ & $\mathrm{~km} 1806$ \\
\hline 10000 & 3,42 & 2,35 & 1,83 \\
\hline 9000 & 3,07 & 2,32 & 1,76 \\
\hline 8000 & 2,90 & 2,29 & 1,68 \\
\hline 7000 & 2,76 & 2,26 & 1,59 \\
\hline 6000 & 2,65 & 2,23 & 1,54 \\
\hline 5000 & 2,53 & 2,21 & 1,47 \\
\hline 4000 & 2,43 & 2,15 & 1,42 \\
\hline 3000 & 2,24 & 2,09 & 1,39 \\
\hline 2000 & 2,08 & 1,97 & 1,36 \\
\hline 1240 & 1,68 & 1,49 & 1,23 \\
\hline
\end{tabular}

IV. - Le transport de débit solide en suspension dans le profil, au km 1876

\begin{tabular}{|c|c|c|c|c|}
\hline ANNÉE & $\begin{array}{c}\text { DËBIT } \\
\text { LIQUIDE } \\
\text { MOYEN ANNULL } \\
\text { A } \\
\text { BRATISLAVA } \\
\\
\left(\mathrm{m}^{3} / \mathrm{s}\right) \\
\end{array}$ & $\begin{array}{l}\text { TURBIDITÉ } \\
\text { MOYENNE } \\
\text { ANNUELLE } \\
\left(\mathrm{g} / \mathrm{m}^{3}\right)\end{array}$ & $\begin{array}{l}\text { TRANSPORT } \\
\text { MOYEN } \\
\text { ANNUEL EN } \\
\text { SUSPENSION } \\
(\mathrm{kg} / \mathrm{s})\end{array}$ & $\begin{array}{c}\text { MASSE } \\
\text { TOTALE DES } \\
\text { MATERIAUX } \\
\text { EN } \\
\text { SUSPENSION } \\
\text { (millions } \\
\text { de t/an) }\end{array}$ \\
\hline 1933 & 1793 & 118,60 & 212,77 & 6,71 \\
\hline 1934 & 1412 & 73,20 & 103,05 & 3,25 \\
\hline 1936 & 2042 & 105,10 & 214,99 & 6,78 \\
\hline 1052 & 1804 & 56,30 & 163,51 & 5,17 \\
\hline 1953 & 1817 & 71,60 & 199,50 & 6,29 \\
\hline 1954 & 1977 & 67,70 & 232,60 & 7,31 \\
\hline 1955 & 2417 & 74,16 & 276,50 & 8,72 \\
\hline 1956 & 2065 & 73,43 & 260,90 & 8,25 \\
\hline 1957 & 2185 & 83,24 & 271,20 & 8,55 \\
\hline 1958 & 2087 & 69,55 & 239,46 & 7,55 \\
\hline 1959 & 2018 & 78,60 & 250,90 & 7,91 \\
\hline 1960 & 1841 & 41,47 & 79,10 & 2,49 \\
\hline Moyenne & 1955 & 75,41 & 208,71 & 6,58 \\
\hline
\end{tabular}

V. - Quelques données relatives aux travanx de régularisation exécutés sur le secteur hongrois-tchécoslovaque du Danube entre 1946 et 195\%.

\begin{tabular}{|c|c|c|c|}
\hline ANNEE & $\begin{array}{c}\text { PierREs } \\
\text { UTiLisées } \\
\left(\mathrm{m}^{3}\right)\end{array}$ & $\begin{array}{c}\text { ReVÈTEMENTS } \\
\text { EXÉcutés } \\
\left(\mathbf{m}^{2}\right)\end{array}$ & $\begin{array}{l}\text { DraGaGES } \\
\text { EFFECTUES } \\
\left(\mathrm{m}^{9}\right)\end{array}$ \\
\hline 1946 & 13631 & - & - \\
\hline 1947 & 25640 & 一 & $\ldots$ \\
\hline 1948 & 45827 & - & - \\
\hline 1949 & 42461 & 22481 & 85536 \\
\hline 1950 & 53973 & 18056 & 8660 \\
\hline 1951 & 54196 & 22503 & 66738 \\
\hline 1952 & 57169 & 4. 176 & 264659 \\
\hline 1953 & 53331 & 6932 & 339284 \\
\hline 1954 & 55741 & 331 & 203819 \\
\hline
\end{tabular}

VI. - Ouelques données relatives aux travaux de régularisation exécutés depuis 1955

\begin{tabular}{|c|c|c|c|}
\hline ANNĚE & $\begin{array}{c}\text { Piernes } \\
\text { UTilisées } \\
\left(\mathrm{m}^{3}\right)\end{array}$ & $\begin{array}{l}\text { REVÊTEMENTS } \\
\text { EXÉCUUTÉs } \\
\left(\mathrm{m}^{2}\right)\end{array}$ & $\begin{array}{c}\text { Dragages } \\
\text { EFFECTUÉS } \\
\left(\mathrm{m}^{3}\right)\end{array}$ \\
\hline 1955 & 57245 & 1290 & 698959 \\
\hline 1956 & 41764 & 223 & 639678 \\
\hline 1957 & 42956 & 2607 & 915781 \\
\hline 1958 & 62169 & 8805 & 1180686 \\
\hline 1959 & 70807 & 1556 & 1085781 \\
\hline 1960 & 57306 & 2116 & 1256762 \\
\hline 1961 & 56059 & 6820 & 1545631 \\
\hline
\end{tabular}

